\title{
Energy and Latency of Beamforming Architectures for Initial Access in mmWave Wireless Networks
}

\author{
C. Nicolas Barati ${ }^{1 *}$, Sourjya Dutta ${ }^{2}$, Sundeep Rangan ${ }^{2}$ and Ashutosh Sabharwal ${ }^{1}$
}

\begin{abstract}
Future millimeter-wave systems, 5G cellular or WiFi, must rely on highly directional links to overcome severe pathloss in these frequency bands. Establishing such links requires the mutual discovery of the transmitter and the receiver, potentially leading to a large latency and high energy consumption. In this work, we show that both the discovery latency and energy consumption can be significantly reduced using fully digital front-ends. In fact, we establish that by reducing the resolution of the fully digital front-ends we can achieve lower energy consumption compared to both analog and high-resolution digital beamforming. Since beamforming through analog front-ends allows sampling in only one direction at a time, the mobile device is "on" for a longer time compared to a digital beamformer, which can get spatial samples from all directions in one shot. We show that the energy consumed by the analog front-end can be four to six times more than that of the digital front-ends, depending on the size of the employed antenna arrays. We recognize, however, that using fully digital beamforming post beam discovery, i.e., for data transmission, is not viable from a power consumption standpoint. To address this issue, we propose the use of digital beamformers with low-resolution analog to digital converters (4 bits). This reduction in resolution brings the power consumption to the same level as analog beamforming for data transmissions while benefiting from the spatial multiplexing capabilities of fully digital beamforming, thus reducing initial discovery latency and improving energy efficiency.
\end{abstract}

Keywords: Millimeter wave, Beamforming, Energy consumption, Beam discovery, Initial access

\section{Introduction}

Almost all of the current wireless communication takes place in a relatively small region of the electromagnetic spectrum below $6 \mathrm{GHz}$. This region has been allocated by government agencies around the world for commercial, civilian, military, public safety, and experimental use. However, the proliferation of devices and services that use or depend on wireless technologies has caused an ever-increasing discrepancy between the demand and the available bandwidth or degrees of freedom (DoF) This discrepancy termed spectrum crunch, if not addressed, will lead to lower data rates and reduced quality of service. Spectrum crunch will become even more acute when Internet of Things ${ }^{39}$ and device-todevice communication traffic are added to the already overloaded networks.

Increasing the DoFs is the only option for the next-generation $(5 \mathrm{G})$ wireless systems. The use of millimeter-wave (mmWave) enables an increase in DoFs by adding more bandwidth due to the availability of large (in the order of a few $\mathrm{GHz}$ ) unlicensed spectrum between 30 and
Degrees of Freedom (DoF) in Wireless: A bandlimited communication signal has $2 W T$ degrees of freedom in the high SNR regime, where $W$ is the bandwidth of the system and $T$ the duration of the transmitted signal. Increasing $\mathrm{W}$ results in the increase of DoFs, in turn resulting into an increase in system's capacity. An alternative way of increasing the DoFs, thus capacity, is to add a spatial dimension: transmit and receive in multiple parallel spatial streams. This is what (massive) MIMO does and has essentially the same effect as increasing the system's bandwidth.

Spectrum Crunch: The scarcity of frequency resources, i.e., spectrum, necessary for wireless communication. This scarcity is the result of demand in wireless services exceeding the available spectrum necessary for accommodating this demand.

${ }^{1}$ Rice University, 6100 Main Street, Houston, TX 77005, USA.

${ }^{2}$ NYU WIRELESS, 370 Jay Street, 9 Floor, Brooklyn, NY 11201, USA *nicobarati@rice.edu 
Pathloss: The loss in signal strength when arriving at the receiver. In the free-space model, it is a function of the distance separating the transmitter and the receiver and the carrier frequency. Signals operating at higher frequencies, such as in mmWave systems, suffer from higher pathloss compared to those in lower ones.

Beamforming: Creating a point of focus in space for the signal to be transmitted to, or received from, an intended set of points rather than omnidirectionally whereby the signal energy is broadcast to every direction equally. Most commonly done using an array of antenna elements.

Initial Access: The process of establishing a link-layer connection between the user equipment and the basestation. It is comprised of several message exchanges involving base-station and user equipment detection, device recognition, and contention resolution in case more than one user equipment attempt access at the same time.
$300 \mathrm{GHz}$. However, as explained by Friis' law ${ }^{41}$, signals transmitted in mmWaves have high isotropic pathloss, i.e., they decay at a much higher rate with the traveled distance. This leads to a reduced communication range compared to sub-6 GHz systems. Furthermore, mmWaves exhibit characteristics resembling the visible light. For example, they have high penetration loss through most material and hence are easily blocked by the surrounding objects. mmWave systems can overcome these shortcomings by employing beamforming (BF) That is, arrays of multiple antenna elements are used to extend the communication range and avoid obstacles in the environment by directing the signal energy in an intended direction.

However, the reliance of mmWave communication on directional links through beamforming poses new challenges that do not exist in wireless systems over the microwave bands. The transmitter $(\mathrm{Tx})$ and the receiver $(\mathrm{Rx})$ must first discover each other directions before they can start the data communication. This process, known in cellular systems as initial access, is generally performed omni-directionally (or using very wide beams) in the sub- $6 \mathrm{GHz}$ bands. But, due to the high path loss, if mmWave systems were to follow the same paradigm, the range of mutual discovery would be much smaller than the range where directional high-rate communication would be possible. Therefore, mutual discovery must be performed in a directional manner.

The directional discovery phase can last for a long time when the base station and the user employ arrays with many antenna elements forming narrow beams. While searching for the base station, the battery-limited user is always "on" burning energy. We show here that this energy consumption can be reduced significantly by employing a low-resolution fully digital frontend on the user side. The reason is, beamforming through a digital front-end reduces the discovery latency (or delay) by an order of magnitude compared to an analog front-end. Hence, the user is "on" for a shorter span of time, leading to considerable energy savings.

While the focus of this paper is on directional discovery in initial access, directional discovery is expected to be triggered in other phases of mmWave communication too. For example, in recovery from link failures, which will be frequent due to the sensitivity of the mmWave links to obstacles and changes in the environment. Handovers to a new base station will also be frequent since mmWave cells will be smaller in size compared to the current ones, and a mobile user may go through more cells for the same distance traveled. More importantly, since the batterydependent devices operate at such high frequencies and bandwidth, more aggressive use of sleep/ idle mode (discontinuous reception) will be critical from an energy consumption standpoint. For each of these operations, the user device must often (re-) discover the direction to the connected and neighboring base station(s). Therefore, it is extremely crucial that directional discovery and beam alignment is fast and energy-efficient.

\subsection{Contributions}

In this paper, we look into the problem of latency and energy consumption in directional discovery in mmWave systems during initial access. Our focus is on $5 \mathrm{G}$ cellular systems where the issue of communication range is more important and challenging than short-range mmWave communication, e.g., 802.11ad WiFi. There are two key take-away points in our work:

1. Digital beamforming results in both low latency and low energy consumption during initial discovery compared to analog beamforming.

2. Employing low-resolution analog to digital converters (ADCs) in fully digital frontends can achieve low latency and even lower power consumption for both control signaling and data transmissions.

Mutual directional discovery requires the transmitter and the receiver search in their surrounding angular space at a granularity inversely proportional to the size of their antenna arrays: larger antenna arrays result in narrower beams potentially leading to higher discovery delays. In a cellular environment, this latency will also affect user handovers between different base stations, alternative link discovery in case of blockage, and the "idle mode" to "on mode" circles.

We show that due to the large latency in directional discovery incurred by analog beamforming, its energy consumption is greater not only than low-resolution digital but also than highresolution digital. This difference in energy consumption increases with the size of the antenna arrays. When a 4-by-4 antenna array is used, analog beamforming burns as much as six times more energy than digital. This is due to the fact that analog beamforming needs more time to 
sample an angular domain that increases in size with the number of antennas.

Leveraging our previous work ${ }^{11,12}$, we establish a relationship between the beamforming architecture and the mutual discovery delay within the context of 3GPP initial access for mmWave networks ${ }^{5}$. Specifically, we show that between analog and digital beamforming, digital outperforms analog by a large margin-in the order of 300-900 ms. Similar to ${ }^{20,21}$, we detail and compare the power consumption of four beamforming architectures, namely, analog, digital, low-resolution digital, and hybrid, by assessing the components and devices they are comprised of. It is known that by reducing the resolution of analog-to-digital converters (ADC) we can significantly reduce the power consumption of a fully digital beamforming circuit (henceforth referred to as low-resolution fully digital). However, we show that while this reduction comes at a penalty of less than $1 \mathrm{~dB}$ SNR in the low SNR regime (up to $10 \mathrm{~dB})^{20}$, the discovery delay is kept at the same low levels as digital beamforming with high resolution-20-80 ms. Thus, low-resolution fully digital beamformers outperform analog as they can be power efficient during both data transmissions as well as signaling control messages.

Interestingly, in most studies related to mmWave systems, analog beamforming has been preferred over digital due to its low device power consumption. However, as we show, when discovery delay in initial access is taken into account, analog beamforming can burn multiple times more energy than any other alternative.

\subsection{Related Work}

Due to the reliance on highly directional links at mmWave frequencies, efficient beam management is key to establishing and maintaining a reliable link. A critical component of beam management is the beam discovery procedure for initial access. Current technical literature assumes an analog or hybrid front-end which limits the number of usable spatial streams. For instance, ${ }^{18,37}$ present a heuristic method to design beam patterns for initial beam discovery for hybrid beamformers. Raghavan et al. ${ }^{40}$ propose to vary the beam widths depending on the user link quality. They show that users with good link (high SNR) can be detected with wide beams leading to a decrease in detection delays. In another direction of research, ${ }^{29,31,53}$ aim at designing optimal beam code books, i.e., sets of directions for connection establishment. Another important area of research has been the use of side information; for instance location information, channel quality measurements at microwave bands, etc., have been studied in ${ }^{6,14,15,19,23,25,34,38,51}$. Moreover, the work in $^{44}$ proposes the use of online machine learning algorithms for beam detection for vehicular communications at mmWave. The works in $^{33,42,54}$ use game- theoretic methods, whereas in $^{27,48}$, the authors use genetic algorithms for initial beam discovery.

$\mathrm{In}^{9}$, the authors present a theoretical analysis of the trade-off between spending resources for accurate beam alignment on the one hand and using them for actual data communication on the other. More interestingly, the analysis in ${ }^{9}$ shows that with large coherence block lengths, exhaustive beam search outperforms hierarchical search. This understanding is reflected in the current $3 \mathrm{GPP}$ specifications ${ }^{2-5}$ on initial access where initial beam discovery and alignment is achieved through exhaustive search. We will discuss the 3GPP new radio beam search procedure briefly in Sect. 2.2. A detailed overview of the beam management procedure for $5 \mathrm{G}$ systems can be found in $^{26}$.

Critical to beam discovery and beam alignment is the efficient signaling of pilot or synchronization signals and channel estimation. To this end, the work in $^{22}$ leverages the sparsity of the mmWave channel. The authors use a compressed sensing framework for estimating the number of measurements necessary for estimating the channel covariance matrix for beam/angle detection with high confidence. Similarly, in ${ }^{47}$ compressed sensing is used for fast angle of arrival/departure and second-order channel statistics estimation. $\mathrm{In}^{52}$, a compressed sensing-based algorithm robust to frequency offsets and phase noise is presented. The sparsity of the mmWave channel is exploited in $^{13}$, where a novel algorithm based on multiple-armed bandit beam selection for both initial beam alignment and beam tracking is proposed. $\mathrm{In}^{28}$, Hashemi et al. exploit the channel correlation to reduce the searching space and subsequently, the delay of beam discovery.

To better understand the interplay between the hardware (and power) constraints at mmWave and the beam discovery delay, in our previous work ${ }^{11,12}$, we presented a comparative analysis of analog and digital beamforming in terms of synchronization signal detectability and delay. We show that digital beamforming, even with low-resolution quantizers, performs dramatically better compared to analog or hybrid. Furthermore, our recent work, ${ }^{20,21}$,
Synchronization signal: A signal transmitted periodically by the base-station. It helps the user equipment to detect neighboring base-station and to get frame-synchronized, i.e., to make out the boundaries of the cell frames in time. 


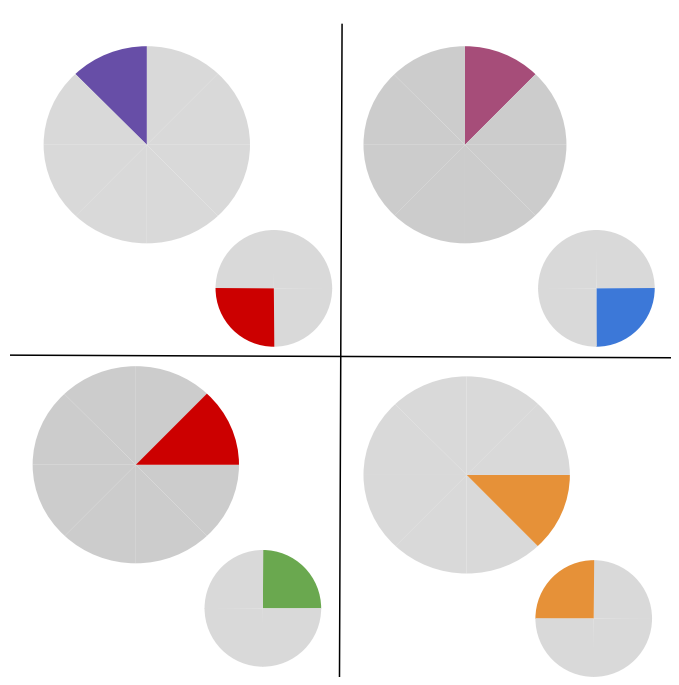

(a) Sectorized Search

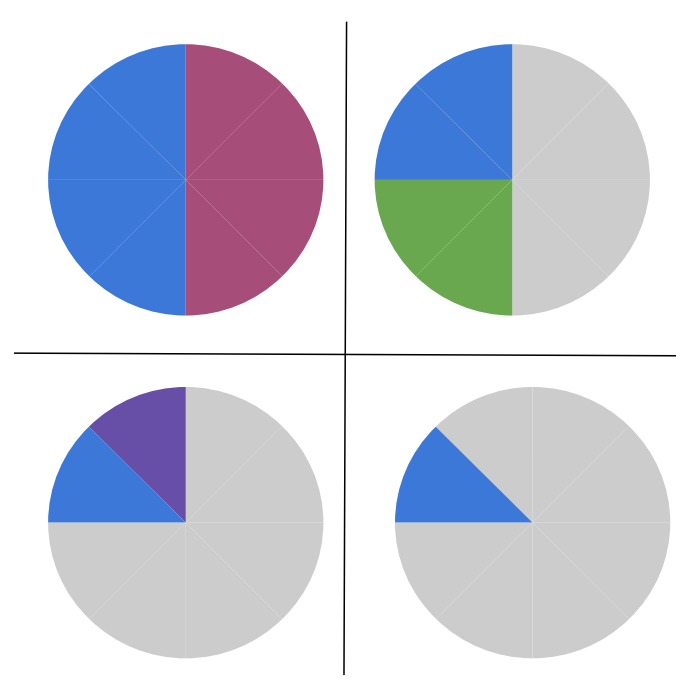

(b) Hierarchical Search

Figure 1: Beam discovery in mmWave bands. Panels read from left to right and top to bottom. a Search over narrow sectors: the receiver, larger shape, and the transmitter, smaller shape, sequentially visit each sector combination until mutual discovery. b Hierarchical search. The receiver gradually narrows its beams in a series of steps.

presents a thorough study of various beamforming architectures at both the transmitter and the receiver sides. There, we show that employing low-resolution digital beamforming introduces negligible loss in the system rate under realistic mmWave cellular assumptions. Based on this, we argue that as low-resolution fully digital front-ends can have low control delays while having the same power efficiency as analog and hybrid beamformers for the data plane. To the best of our knowledge, this is the first work where energy consumption during initial beam discovery has been studied. In this work, we show that a low-resolution fully digital beamformer is energy-efficient in both control plane in general, initial access in particular, and data plane.

The rest of the paper is organized as follows. In Sect. 2 we give an overview of the beam discov-

3GPP New Radio (NR): The set of $5 \mathrm{G}$ system specifications 3GPP members have agreed upon. It defines among other things waveforms, frame and sub-frame structure, modulation and coding schemes. ery problem and present the $3 \mathrm{GPP}$ new radio (NR) discovery procedure. In Sect. 3, we present the system model. We derive a correlation-based detector for beam discovery, present four mmWave beamformers and comment on the power consumption of each one of them. In Sect. 4, we evaluate through simulations the performance of the analog and high/low-resolution beamformers in terms of discovery delay and energy consumption.

Notation We use small boldface letters (h) to denote vectors and boldface capital letters $(\mathbf{H})$ for matrices. Conjugate and conjugate transpose are denoted with $\mathbf{h}^{*}$ and the $\mathrm{L}-2$ norm with $\|\mathbf{h}\|$ and $\|\mathbf{H}\|$ for vectors and matrices, respectively.

\section{Beam Discovery Through Beamsweep}

Consider a transmitter-receiver pair operating in mmWave bands. Since they communicate through directional links, they both must discover each other in the spatial domain before the data communication can begin. An intuitive approach to this problem is to divide the spatial domain around the transmitter (or receiver) into multiple non-overlapping sectors, as presented in Fig. 1a. Each of these sectors corresponds to a transmitter or receiver beam. All possible combinations of the pair of transmitter-receiver beams are termed as the beamspace. Increasing the number of antennas makes the beams narrower, which increases the beamforming gain but also makes the size of the beamspace larger. This poses a fundamental problem for mmWave systems. On the one hand, narrow beams are necessary for usable link budgets. On the other, beam discovery, i.e., finding the two matching beam pairs at the transmitter and the receiver, a difficult problem.

In this work, we assume a stand-alone communication model where the transmitter and the receiver are not assisted by out-of-band information regarding timing or position. The non-stand-alone model has been investigated in $^{6,14,15,19,23,25,34,38,51}$ and is not discussed in this work. There are two main approaches to beam 
discovery/selection under this assumption. One, called beamsweep, requires both the transmitter and the receiver to exhaustively search over the entire beamspace by measuring the received power for every possible transmitter-receiver beam pair. In another, the receiver side starts listening on the channel with the widest possible beam and step by step converges to the narrowest one. This is called hierarchical search, see Fig. $1 b$.

Both these techniques assume that a known signal called the synchronization signal in cellular is transmitted periodically, and the receiver will have to determine the direction in the beamspace where the incoming signal is stronger. While hierarchical search in principle is superior to beamsweep in terms of search delay ${ }^{17}$, mmWave standards for both WiFi and cellular have adopted a beamsweep-based procedure due to its simplicity ${ }^{2-5,30}$. In this work, we will also follow the beamsweep paradigm and our analysis and results are derived based on this assumption.

\subsection{Effect of Beamforming on the Beamspace}

Under the beamsweep assumption, the effective size of the beamspace is a function of the beamforming scheme employed at the transmitter and the receiver. That is, the beamforming scheme dictates how many directions the receiver can inspect in a single observation. There are three beamforming schemes:

Digital beamforming In this architecture, each antenna element is connected to a radio frequency (RF) chain and a pair of data converters, analog-to-digital or digital-to-analog converters (ADC or DAC). The beamforming (or spatial filtering) is performed by the digital baseband processor. For wideband systems with a large number of antennas, this architecture can have high power consumption when high precision DACs and ADCs are used. One way to use digital beamformers at mmWave is to use DACs and ADCs with few bits of quantizer resolution. This is attractive for beamformed systems as having digital samples from every antenna element allows the receiver to inspect as many directions as the number of antenna elements with full directional gain using only one observation of the channel. This reduction of the number of needed observations becomes significant as the beamspace grows and can potentially reduce the total power consumed during the search procedure.
Analog beamforming To avoid the use of a large number of DACs and ADCs, analog beamformers perform beamforming (or spatial filtering) on the analog (in RF or intermediate frequency) signal using RF phase shifters and power combiners (or splitters). The use of just a pair of ADCs considerably reduces the power consumed by these frontends and hence, these are considered a prime candidate for initial mmWave-based cellular devices. However, analog beamformers can point their beams only in one direction at a given time. This leads to potentially high delays when the beamspace is large.

Hybrid beamforming This scheme is a combination of the digital and analog beamforming. A part of the beamforming is performed by $M$ analog RF beamformers. These beamformed signals are digitized and combined (or precoded) by the digital baseband circuitry. This allows the receiver to inspect $M$ directions at each time instance. Now, at an extreme $M=1$, where we have analog beamforming. At the other extreme, $M$ equals the number of antenna elements, where the scheme is effectively digital beamforming. Choosing $M$ trades off spatial multiplexing capabilities on the one hand and energy consumption on the other.

Consider a transmitter equipped with an antenna array comprised of $N_{\mathrm{Tx}}$ antenna elements and a receiver equipped with $N_{\mathrm{Rx}}$ elements. This means that the size of the beamspace is equal to the product of $N_{\mathrm{Tx}}$ by $N_{\mathrm{Rx}}$. If they both use analog beamforming, then they must visit all these directions at $N_{\mathrm{Tx}} \times N_{\mathrm{Rx}}$ separate channel inspections. Hence, the effective size of the beamspace is,

analog: $\quad L_{\mathrm{an}}=N_{\mathrm{Tx}} \times N_{\mathrm{Rx}}$.

Now, suppose that the transmitter still uses analog beamforming but the receiver uses digital. Then, the receiver can "listen" on all the $N_{\text {Tx }}$ directions simultaneously and hence in this effective case the size of the beamspace becomes

digital: $\quad L_{\mathrm{di}}=N_{\mathrm{Tx}}$.

Applying the same logic, for hybrid beamforming, it is easy to see that the effective size of the beamspace is

hybrid: $\quad L_{\mathrm{hy}}=\frac{N_{\mathrm{Tx}} \times N_{\mathrm{Rx}}}{M}$ 
Other combinations of beamforming schemes on either the transmitter or the receiver yield an effective beamspace of various sizes. Adopting one beamforming scheme versus the other has a fundamental impact on the effective beamspace. This, in turn, affects the time needed for the receiver to determine the best direction of the incoming signal. Furthermore, during beamsweeping the receiver front-end is always on. A front-end architecture that can listen on one or a few directions at a time will hence need to be powered on for a longer period of time to measure all the possible beam pairs. This can mean a considerable increase in the effective power consumed by analog and hybrid beamformers. In the next sections, we quantify the impact of the size of the beamspace and the choice of the beamformers on the energy consumed by the beam discovery procedure.

\subsection{The 3GPP NR Paradigm}

As an illustrative example of a system using beamsweep, we will discuss the 3GPP NR physical layer standard for initial access (IA) in mmWave bands, 3GPP frequency range 2 (FR2). Our analysis and results in the upcoming sections will all have an NR system as an underlying assumption. We chose NR for two reasons. One, it is the standard defined for $5 \mathrm{G}$ cellular systems, so it is expected to be adopted by millions of devices in the coming years. Two, the NR standard offers a well-defined set of assumptions regarding the beam discovery process and time and frequency numerology. This allows us to evaluate the different beamforming schemes with respect to energy consumption within a widely accepted context.

We next present the beam discovery process which is one of the basic steps of NR initial access, i.e., the procedure of establishing a linklayer connection between a base station, referred to as NR 5G nodeB (gNB) in the NR standard, and a mobile device or user equipment (UE).

\subsubsection{NR Beam Discovery}

Initial access involves a set of message exchanges between the gNB and the user equipment, whereby the user equipment identifies a serving gNB and synchronizes to it. The user equipment learns the physical cell identity of the gNB, sends back its own ID, and finally attaches to the cell. In this work, we will focus only on the first part of NR initial access: beam and cell discovery on the user equipment side, since this is the most critical in terms of detection delay and energy consumption.

On the gNB side, all the $N_{\text {Tx }}$ directions are swept with a periodicity of $T=20 \mathrm{~ms}$. More specifically, every $T$ and for an interval of duration $T_{\mathrm{ssb}}=5 \mathrm{~ms}$, the gNB transmits $B$ blocks of four OFDM symbols in $N_{\text {TX }}$ directions. These transmissions during $T_{\text {ssb }}$ are called a synchronization signal (SS) burst and each block of the four OFDM symbols, an SS block. Each SS block is comprised of the primary synchronization signal (PSS), the secondary synchronization signal (SSS) and the physical broadcast channel $(\mathrm{PBCH})$. The PSS and the SSS together make up the physical ID of the cell. The first one takes one of three possible values among $\{0,1,2\}$ while the latter, one of $\{0,1, \ldots, 335\}$, resulting in a total of 1008 unique cell IDs. Each of these signals takes up 127 subcarriers in frequency, while an entire SS block, including $\mathrm{PBCH}$, includes 240 sub-carriers. In Fig. 2, we depict the time-frequency resources occupied by the SS block within an NR frame, subframe and slot.

Figure 3 shows an example of several SS blocks within a single SS burst. For the mmWave bands, the NR standard provisions a total of $B=32$ SS blocks during each SS burst. It is assumed that each SS block is transmitted simultaneously in two directions using hybrid beamforming. Hence, a mmWave gNB can support up to 64 non-overlapping directions. As an example, in Fig. 4, we depict a scenario of a gNB beamsweeping $N_{\mathrm{Tx}}=8$ directions using $B=4$ SS blocks per SS bursts.

Now, the user equipment is also sweeping $N_{\mathrm{Rx}}$ directions in searching for SS burst, see Fig. 4. These signals are known to the user equipment. It has to determine which one of the possible three PSS sequences and 336 SSS sequences were sent. Since the structure of an SS block is also known, once the PSS is detected and the optimal direction is found, the user equipment will move on to detecting the SSS within the same SS block, which is the third OFDM symbol, as shown in Fig. 2. Thus, the most critical part of beamsweeping is the detection of the PSS which unlocks all the remaining steps of initial access.

The user equipment is assumed to use analog beamforming. Hence, unlike the gNB it can probe only one direction at a time. Since the gNB sends the SS blocks in two directions simultaneously, according to Sect. 2.1 the effective size of the beamspace around the gNB and the user equipment is $L=\left(N_{\mathrm{TX}} \times N_{\mathrm{RX}}\right) / 2$. 


\section{Frame (10ms)}

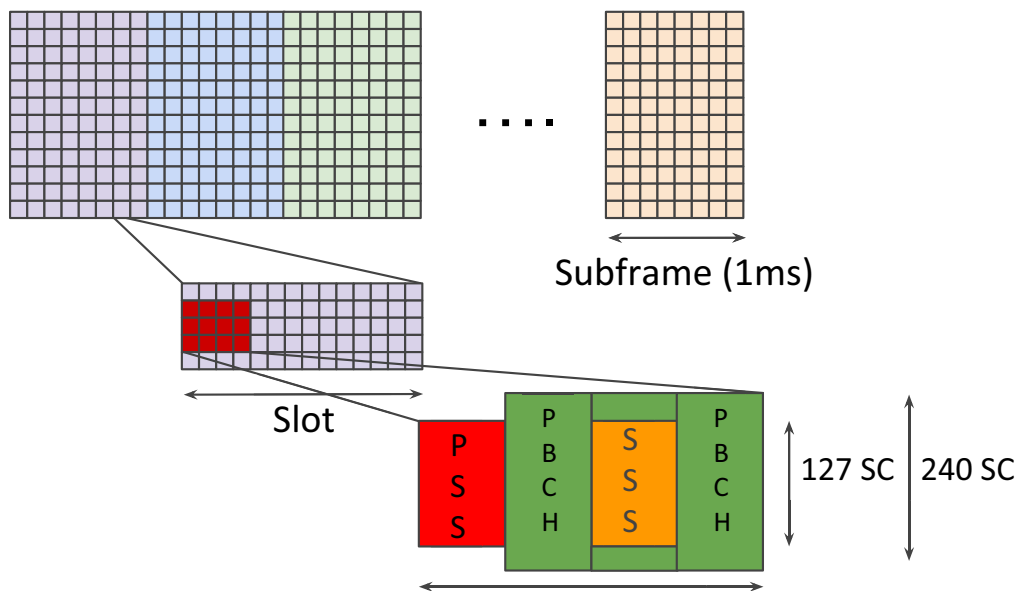

SS block

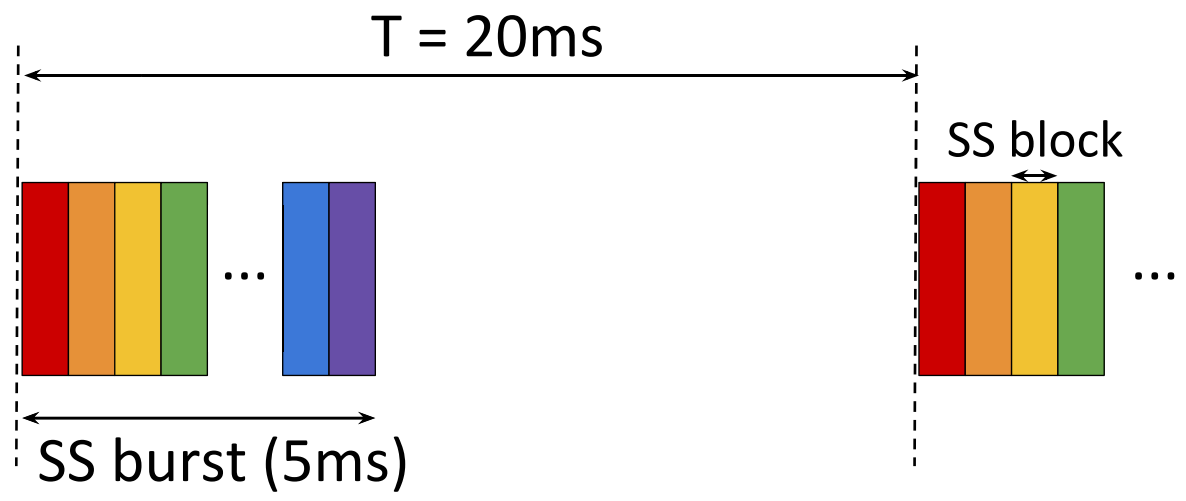

Figure 3: SS burst. Each burst lasts for $5 \mathrm{~ms}$ and is repeated every $20 \mathrm{~ms}$. Each SS block within an SS burst is shown in a different color.

\section{Signal and System Model}

We will leverage the NR beam discovery process described earlier for our analysis and modeling, with a few simplifying assumptions. We consider a single cell of radius $d_{r}$ with the gNB situated at the center transmitting the PSS signals periodically. The user equipment, through analog beamforming will sweep $N_{R x}$ directions sequentially in search of the PSS signal.

Our first simplification is assuming analog beamforming at the gNB. Therefore, the user equipment and the gNB together will have to sample a beamspace of size $L_{\mathrm{ang}}=N_{\mathrm{tx}} \times N_{\mathrm{rx}}$.
Note that for user equipment at low SNR, i.e., those at the edge of the cell, it may be needed to cycle through the beamspace more than once. We will denote each cycle with $k=1,2, \ldots, K$.

Our next assumption is that similar to ${ }^{12}$, the dominant path between the gNB and the user equipment is a line of sight (LOS) path aligned with exactly one of the transmitter-RX directions/sectors $\ell^{*}$ in the beamspace. Although this assumption may seem unrealistic, since real channels are seldom comprised of a single path, it is only used to derive our detectors below. In 


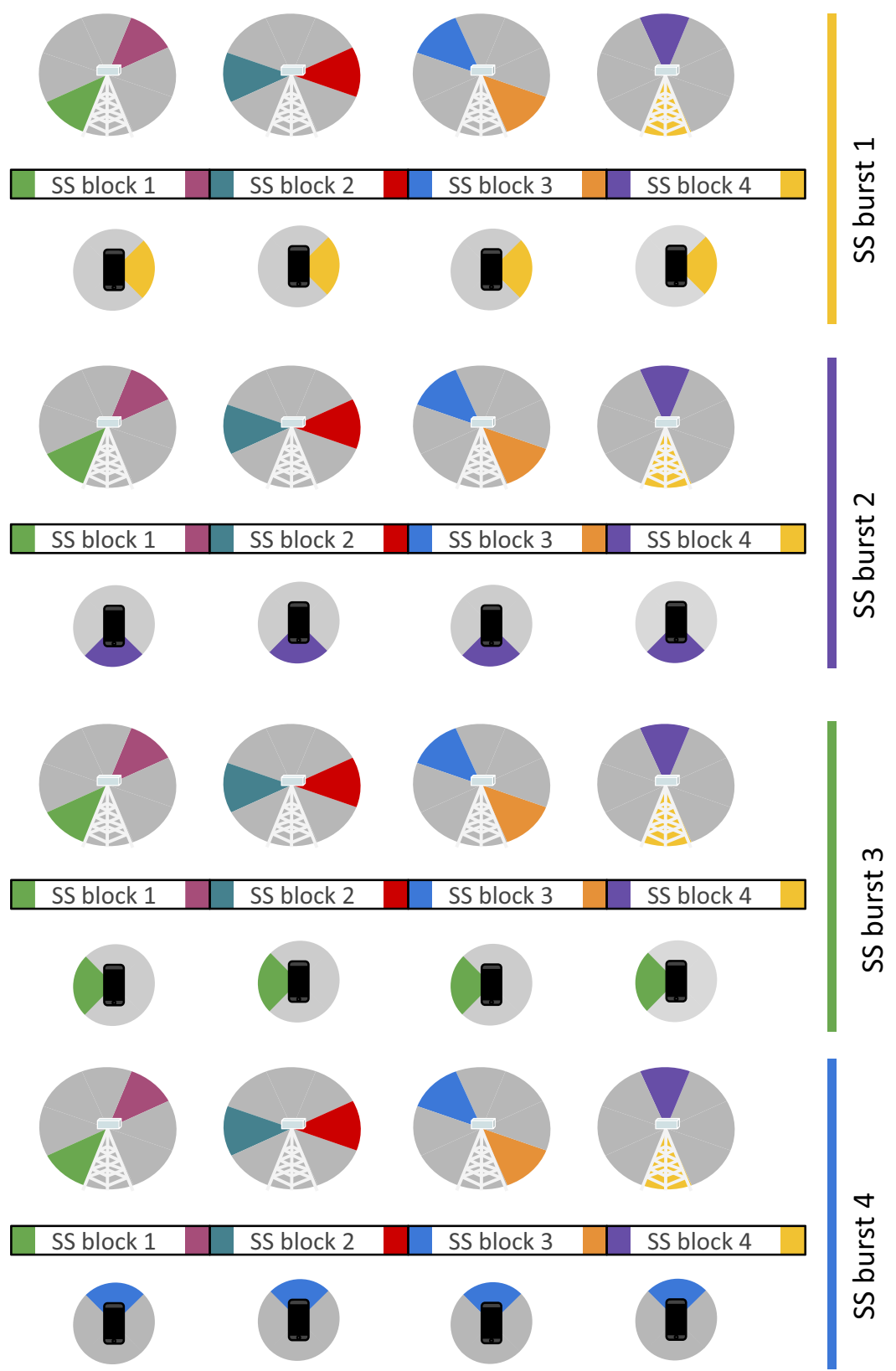

Figure 4: Example of beam discovery within four SS bursts. During each SS burst, the gNB covers $N_{T x}=8$ directions in 4 SS blocks. The user equipment, on the other hand, scans all the $N_{R x}=4$ directions in four SS bursts. Thus, the user equipment and the gNB together need four SS bursts to cover the beamspace of size $L=16$.

our evaluation and simulations, we will test our detectors using a channel model derived from real measurements ${ }^{8}$.

With these assumptions in mind, we model the received post-analog-BF received complex signal $\mathbf{y}_{k \ell}$ at the user equipment during the $\ell$ th sampling of the beamspace and $k$ th sweeping cycle as: $\mathbf{y}_{k \ell}=\mathbf{u}_{k \ell}^{*} \mathbf{H}_{k \ell} \mathbf{v}_{k \ell}^{*} \mathbf{x}+\mathbf{n}_{k \ell}, \quad \mathbf{n}_{k \ell} \sim \mathcal{C N}\left(0, \sigma_{k \ell}^{2} \mathbf{I}_{D}\right)$.

The transmitted PSS signal $\mathbf{x} \in \mathbb{C}^{D}$ is in a signal space with $D \approx T_{\mathrm{PSS}} \times W_{\mathrm{pss}}$ orthogonal degrees of freedom, where $T_{\mathrm{PSS}}$ and $W_{\mathrm{pss}}$ are the PSS duration in time and bandwidth occupied by the PSS signal, respectively. We assume the PSS signal to be unit norm, $\|\mathbf{x}\|=1$. The vectors $\mathbf{u}_{k \ell} \in \mathbb{C}^{N_{\mathrm{RX}}}$ and $\mathbf{v}_{k \ell} \in \mathbb{C}^{N_{\mathrm{TX}}}$ are, respectively, the 
user equipment and gNB side beamforming vectors along a transmitter-receiver direction $\ell$ in the sectorized beamspace at the $k$ th sweep cycle. They are assumed to be of fixed norm.

The MIMO channel $\mathbf{H}$ is assumed to be flat fading within the PSS bandwidth and constant in each sweeping cycle our null hypothesis, $H_{0}$, is that the signal is not present. Conversely, our alternative hypothesis, $H_{1}$, is that the signal is present, i.e., the user equipment is probing the direction in which the gNB is transmitting. Hence, Eq. (4) under $H_{0}$ and $H_{1}$ becomes

$$
\begin{aligned}
& H_{0}: \mathbf{y}_{k \ell}=\mathbf{n}_{k \ell} \\
& H_{1}: \mathbf{y}_{k \ell}=\alpha_{k \ell^{*}} \mathbf{x}+\mathbf{n}_{k \ell^{*}} \quad \text { (signal present at correct beamspace direction), }
\end{aligned}
$$

over the PSS transmission time $T_{\mathrm{PSS}}$. It is defined as

$$
\mathbf{H}=h_{k \ell} \mathbf{u}_{\ell^{*}} \mathbf{v}_{\ell^{*}}^{*},
$$

where $h_{k \ell}$ is a small-scale fading coefficient. The vectors $\mathbf{u}_{\ell^{*}}$ and $\mathbf{v}_{\ell^{*}}$ are the spatial signatures of the user equipment and the gNB antenna arrays describing a single LOS path between them aligned with only one of the transmitter-RX directions in the beamspace. They are given as

$$
\begin{aligned}
& \mathbf{u}_{\ell^{*}}=\left[1, e^{-j 2 \pi \Delta \cos \left(\phi_{\ell^{*}}^{\mathrm{Rx}}\right)}, \ldots, e^{-j 2 \pi \Delta\left(N_{\mathrm{RX}}-1\right) \cos \left(\phi_{\ell^{*}}^{\mathrm{Rx}}\right)}\right]^{\mathrm{T}} \\
& \mathbf{v}_{\ell^{*}}=\left[1, e^{-j 2 \pi \Delta \cos \left(\phi_{\ell^{*}}^{\mathrm{Tx}}\right)}, \ldots, e^{-j 2 \pi \Delta\left(N_{\mathrm{TX}}-1\right) \cos \left(\phi_{\ell^{*}}^{\mathrm{Tx}}\right)}\right]^{\mathrm{T}},
\end{aligned}
$$

where $\phi_{\ell^{*}}^{\mathrm{Rx}}$ and $\phi_{\ell^{*}}^{\mathrm{Tx}}$ are the angles of arrival and departure in the directions of $\ell^{*}$ and $\Delta$ is the distance between elements of the antenna arrays measured in wavelengths. Finally, $\mathbf{n}$ in (4) is the i.i.d. complex additive white Gaussian noise vector with covariance $\sigma_{k \ell}^{2} \mathbf{I}_{D}$ and $\mathbf{I}_{D}$ is the $D \times D$ identity matrix.

The aim of the user equipment and the gNB is to mutually steer their beams along their spatial signatures defined in (6). That is, to apply beamforming vectors, $\mathbf{u}_{\ell}$ and $\mathbf{v}_{\ell}$, as close to $\mathbf{u}_{\ell^{*}}$ and $\mathbf{v}_{\ell^{*}}$ as possible. This, based on our models in Eqs. (4) and (5) and our fixed norm assumption over the beamforming vectors, leads to the maximization of the energy of the received signal vector $\mathbf{y}_{k \ell}$.

\subsection{Signal Detection}

Given the signal and transmission model above we now derive the PSS detector through hypothesis testing. We will follow the same line of analysis given $\mathrm{in}^{12}$. Since we have assumed that the dominant path from the gNB to the user equipment is along a single transmitter-receiver beamspace direction $\ell^{*}$ (here the asterisk denotes the true or best direction), for each probed direction where the scalar channel coefficient $\alpha_{k \ell^{*}}$ is the result of applying the beamforming vectors along the spacial signatures of the user equipment and the gNB and is given by

$\alpha_{k \ell^{*}}=\mathbf{u}_{\ell^{*}}^{*} \mathbf{H}_{k \ell^{*}} \mathbf{v}_{\ell^{*}}=h_{k \ell^{*}} \mathbf{u}_{\ell^{*}}^{*} \mathbf{u}_{\ell^{*}} \mathbf{v}_{\ell^{*}}^{*} \mathbf{V}_{\ell^{*}}$.

We define the probability density of the received signal $y$ under the two hypotheses as $p\left(\mathbf{y} \mid H_{i}, \boldsymbol{\alpha}, \sigma^{2}, \ell^{*}\right)$ for $i=\{0,1\}$, where the set $\mathbf{y}$ contains all the observed signals $\mathbf{y}_{k \ell}$ in $K$ beam sweeps. The model contains several unknown parameters, namely, $\boldsymbol{\alpha}$ the set of all the channel coefficients $\alpha_{k \ell^{*}}$, and $\sigma^{2}$ the set of all noise power levels.

Due to these unknown parameters, we will rely on the widely used generalized likelihood ratio test (GLRT) method ${ }^{49}$. The GLRT takes the likelihood distribution under each hypothesis maximized with respect to the unknown parameters and compares their ratio to a threshold. Alternatively, we can use the minimum log-likelihoods of the signal distribution under each hypothesis. These are given as

$$
\begin{aligned}
& \Lambda_{0}:=\min _{\boldsymbol{\sigma}^{2}}-\ln p\left(\mathbf{y} \mid H_{0}, \boldsymbol{\sigma}^{2}\right) \\
& \Lambda_{1}:=\min _{\boldsymbol{\sigma}^{2}, \boldsymbol{\alpha}}-\ln p\left(\mathbf{y} \mid H_{1}, \boldsymbol{\sigma}^{2}, \boldsymbol{\alpha}\right) .
\end{aligned}
$$

We then use the test

$\Lambda:=\Lambda_{0}-\Lambda_{1} \underset{H_{0}}{\stackrel{H_{1}}{\gtrless}} \theta$

for a threshold $\theta$. We observe that under the assumption that the noise vectors are independent in different measurements,

$\ln p\left(\mathbf{y} \mid H_{0}, \sigma^{2}, \ell^{*}\right)=\sum_{k=1}^{K} \sum_{\ell=1}^{L} \ln p\left(\mathbf{y}_{k \ell} \mid \sigma_{k \ell}^{2}, \ell^{*}\right)$
Spatial signature: It is the vector response of the receiver's antenna array to incoming signal.

Generalized likelihood ratio test (GLRT): It is a hypothesis testing tool used for composite hypotheses, i.e., when there are more than one parameters to be estimated. GLRT compares the ratio of the maximum, with respect to the estimation parameters, likelihood functions of each hypothesis to a threshold. Based on this comparison, it outputs the most likely hypothesis.

Hypothesis testing: A statistical method of inference where the plausibility of the observed data coming from a particular distribution, or hypothesis, is tested. 
$\ln p\left(\mathbf{y} \mid H_{1}, \sigma^{2}, \boldsymbol{\alpha}, \ell^{*}\right)=\sum_{k=1}^{K} \sum_{\ell=1}^{L} \ln p\left(\mathbf{y}_{k \ell} \mid \sigma_{k \ell}^{2}, \alpha_{k \ell^{*}}, \ell^{*}\right)$

Therefore, the negative log-likelihoods in Eq. (9) can be re-written as

$$
\begin{aligned}
& \Lambda_{0}=\sum_{k=1}^{K} \sum_{\ell=1}^{L} \Lambda_{0}^{k \ell}\left(\ell^{*}\right) . \\
& \Lambda_{1}=\sum_{k=1}^{K} \sum_{\ell=1}^{L} \Lambda_{1}^{k \ell}\left(\ell^{*}\right),
\end{aligned}
$$

where $\Lambda_{0}^{k \ell}\left(\ell^{*}\right)$ and $\Lambda_{1}^{k \ell}\left(\ell^{*}\right)$ are the minimum negative log-likelihoods in each measurement and given as:

$\Lambda_{0}^{k \ell}\left(\ell^{*}\right)=\min _{\sigma^{2}}-\ln p\left(\mathbf{y}_{k \ell} \mid \sigma_{k \ell}^{2}, \ell^{*}\right)$.

$\Lambda_{1}^{k \ell}\left(\ell^{*}\right)=\min _{\sigma^{2}, \alpha_{k \ell}}-\ln p\left(\mathbf{y}_{k \ell} \mid \sigma_{k \ell}^{2}, \alpha_{k \ell}, \ell^{*}\right)$.

Since the received signal in our model, given by Eq. (7), is Gaussian conditional on the parameters, hence

$-\ln p\left(\mathbf{y}_{k \ell} \mid \sigma_{k \ell}^{2}, \ell^{*}\right)=\frac{1}{\sigma_{k \ell}^{2}}\left\|\mathbf{y}_{k \ell}\right\|^{2}+D \ln \left(\pi \sigma_{k \ell}^{2}\right)$

under the $H_{0}$ hypothesis, and

$$
\begin{aligned}
& -\ln p\left(\mathbf{y}_{k \ell} \mid \sigma_{k \ell}^{2}, \alpha_{k \ell^{*}}, \ell^{*}\right) \\
& =\frac{1}{\sigma_{k \ell}^{2}}\left\|\mathbf{y}_{k \ell}-\alpha_{k \ell^{*}} \mathbf{x}\right\|^{2}+D \ln \left(\pi \sigma_{k \ell}^{2}\right)
\end{aligned}
$$

under the $H_{1}$ hypothesis. Now, we use the above Eqs. (14) and (15) to obtain estimates of the unknown variables which are their minimizers.

We first take $\Lambda_{1}^{k \ell}\left(\ell^{*}\right)$. The channel coefficient $\alpha_{k \ell^{*}}$ that minimizes the log negative likelihood when the signal is present is given as

$\widehat{\alpha}_{k \ell^{*}}=\frac{\mathbf{x}^{*} \mathbf{y}_{k \ell}}{\|\mathbf{x}\|^{2}}$.

Next we move on to minimize over the variance $\sigma^{2}$, which occurs at

$\widehat{\sigma}_{k \ell}^{2}=\frac{1}{D}\left(\left\|\mathbf{y}_{k \ell}\right\|^{2}-\frac{\left|\mathbf{x}^{*} \mathbf{y}_{k \ell}\right|^{2}}{\|\mathbf{x}\|^{2}}\right)$.

When the signal is not present, i.e., $\ell \neq \ell^{*}$, we minimize $\Lambda_{0}^{k \ell}\left(\ell^{*}\right)$ over the variance $\sigma^{2}$. The minimum is obtained at $\widehat{\sigma}_{k \ell}^{2}=\frac{\left\|\mathbf{y}_{k \ell}\right\|^{2}}{D}$.

Substituting Eqs. (16), (17) and (18), in Eq. (13) we obtain

$\Lambda_{0}^{k \ell}\left(\ell^{*}\right)=D \ln \left(\frac{\pi e}{D}\left\|\mathbf{y}_{k \ell}\right\|^{2}\right)$

$\Lambda_{1}^{k \ell}\left(\ell^{*}\right)=D \ln \left(\frac{\pi e}{D}\left(\left\|\mathbf{y}_{k \ell}\right\|^{2}-\frac{\left|\mathbf{x}^{*} \mathbf{y}_{k \ell}\right|^{2}}{\|\mathbf{x}\|^{2}}\right)\right)$.

Combining (19) and from the test (10), and Eq. (12) we have the following log-likelihood difference

$\Lambda=\sum_{k}^{K} \sum_{\ell}^{L}-D \ln \left(1-\rho_{k \ell}\right)$

where the $\rho_{k \ell}$ is the normalized energy of the correlation of the known signal with the received signal, obtained as

$\rho_{k \ell}=\frac{\left|\mathbf{x}^{*} \mathbf{y}_{k \ell}\right|^{2}}{\|\mathbf{x}\|^{2}\left\|\mathbf{y}_{k \ell}\right\|^{2}}$.

This, not surprisingly, means that while the user equipment probes each direction in $K$ beamsweeps, it should correlate the incoming signal with the local replica of the known signal. Then, since Eq. (20) is an increasing function of $\rho_{k \ell}$, and due to our assumption that the signal is present only in one direction within the beamspace, the best direction $\widehat{\ell}^{*}$ is the one in which the correlation was the highest, that is,

$\widehat{\ell}^{*}=\underset{\ell=1, \ldots, L}{\arg \min } \sum_{k}^{K} \sum_{\ell}^{L} \ln \left(1-\rho_{k \ell}\right)$.

While the above detector was derived for analog beamforming under favorable beamspace assumptions, it can also be used for both hybrid and digital beamforming. The difference is that with these beamforming architectures, the receiver has access to $N_{\mathrm{RF}}>1 \mathrm{RF}$ chains and can essentially operate as $N_{\text {RF }}$ parallel analog systems. In the digital beamforming case $N_{\mathrm{RF}}$ is equal to $N_{\mathrm{Rx}}$. Note that with a fully digital detector, the receiver can test all the angles, including those not perfectly aligned in the beamspace. However, for the sake of simplicity and better comparison with analog beamforming, we will not consider a more powerful detector but assume that in digital beamforming too the arrival angles are aligned with the beamspace directions. 


\subsection{RF Architectures and Power \\ Consumption}

While standards documents on the implementation of mmWave cellular systems assume user equipments with analog beamforming and only a single RF chain, we believe that there is much value in using fully digital beamforming. To make our case more concrete in terms of energy consumption, we give here an overview of the receiver's mmWave front-end. Note that the part of the mmWave front-end before the variable gain amplifier (VGA) and the ADCs (see Figs. 5, 6, 7) is the radio frequency front-end (RFFE). We model the power consumption of analog, hybrid, and fully digital and fully digital with low-resolution quantization front tends.

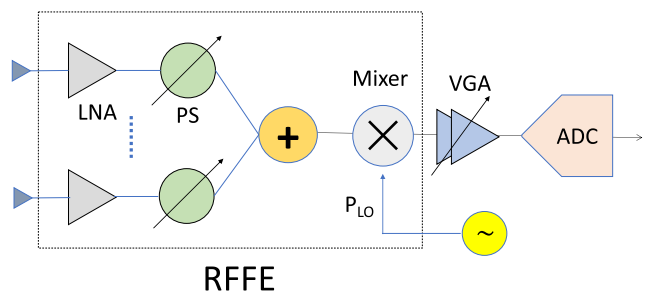

Figure 5: Analog beamforming: receiver frontend architecture.

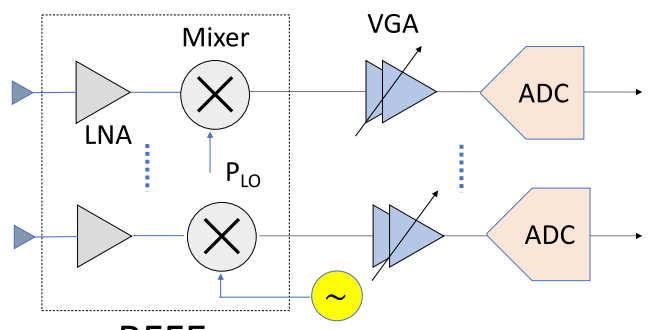

RFFE

Figure 6: Digital beamforming: receiver front-end architecture.

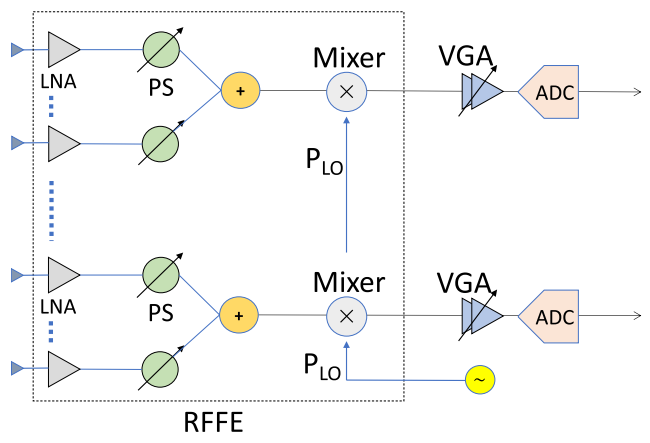

Figure 7: Sub-array hybrid beamforming: receiver front-end architecture.

\subsection{1 mmWave Front-Ends}

Analog front-end Consider the mmWave receiver's analog front-end shown in Fig. 5. It is comprised of $N_{\mathrm{RX}}$ low noise amplifiers (LNA)s, $N_{\mathrm{RX}}$ phase shifter (PS), a mixer, a combiner and a pair of analog-to-digital converter (ADC). The D.C. power consumption of each LNA, $P_{\mathrm{DC}}^{\mathrm{LNA}}$ is a function of its gain $G_{\mathrm{LNA}}^{\mathrm{PS}}$, the noise figure $N_{\mathrm{LNA}}$ and the figure of merit (FoM) and is given as s, $21,46^{2}$

$P_{\mathrm{LNA}}^{\mathrm{DC}}=\frac{G_{\mathrm{LNA}}^{\mathrm{PS}}}{\operatorname{FoM}\left(N_{\mathrm{LNA}}-1\right)}$,

in linear scale.

The PS and mixer are considered to be passive elements. While they do not burn any power, they do, however, introduce insertion losses (IL), which need to be compensated for. For example, the LNA gain above needs to be high enough to offset the loss introduced by the phase shifter ILPS. That is, if an LNA in a circuit without any PS had a gain $G_{\mathrm{LNA}}$, then $G_{\mathrm{LNA}}^{\mathrm{PS}}=G_{\mathrm{LNA}}+$ ILPS.

For the ADCs, power consumption is a function of the sampling frequency $F_{\mathrm{s}}$, ADC's figure of merit and the resolution in bits:

$P_{\mathrm{ADC}}=\mathrm{FoM} \times F_{\mathrm{s}} \times 2^{q}$,

where $q$ is the number of resolution bits. To keep the variable incoming signal within the ADCs dynamic range while keeping a constant baseband power $P_{\mathrm{BB}}^{\text {out }}$ it is necessary to apply gain control to the input of the ADC. This is performed by the variable gain amplifier (VGA) with a gain range between 0 and $G_{\mathrm{VGA}}^{\max }$. For the analog beamforming case this maximum gain is calculated as:

$$
\begin{aligned}
G_{\mathrm{VGA}}^{\max }= & P_{\mathrm{BB}}^{\text {out }}-10 \log \left(N_{\mathrm{RX}}\right)+\mathrm{IL}_{\text {mix }} \\
& -\left(G_{\mathrm{LNA}}^{\mathrm{PS}}-\mathrm{IL}_{\mathrm{PS}}\right)-\mathrm{P}_{\mathrm{RX}}\left(\mathrm{d}=\mathrm{d}_{\max }\right),
\end{aligned}
$$

where $\mathrm{IL}_{\text {mix }}$ is the insertion loss introduced by the mixer and $P_{\mathrm{RX}}\left(d=d_{\max }\right)$ is the maximum distance between the transmitter and the receive, i.e., the cell edge.

Given the above gain, the D.C. power draw of the VGA is given as

$P_{\mathrm{VGA}}^{\mathrm{DC}}=\frac{G_{\mathrm{VGA}}^{\mathrm{max}} F_{\mathrm{BW}}}{\text { FoM } A_{\text {chip }}}$,

where FoM is the FoM of the VGA, $F_{\mathrm{BW}}$ the bandwidth of the VGA in $\mathrm{GHz}$ and $A_{\text {chip }}$ is the active area in $\mathrm{mm}^{2}$. For our analysis we assume that $F_{\mathrm{BW}}=F_{\mathrm{s}}$, i.e., the bandwidth of the VGA is equal to the sampling rate of the ADC.
Radio Frequency Front End (RFFE): The circuitry at the transceiver that processes the radio frequency (RF) signal. This is the circuit between the antenna input/output port and the output/input port of the DAC/ADC.

Quantization: The process digitizing an analog signal. In communication systems, it is done through analog to digita converters (ADCs) where the continuous amplitude of the analog signal is mapped to a finite set of levels. 
Based on the above, the total power consumption of an analog mmWave front-end can be calculated as

$P_{\mathrm{an}}=N_{\mathrm{RX}} P_{\mathrm{LNA}}^{\mathrm{DC}}+P_{\mathrm{LO}}+P_{\mathrm{ADC}}+P_{\mathrm{VGA}}^{\mathrm{DC}}$,

where $P_{\mathrm{LO}}$ is the power draw of the local oscillator.

Digital front-end Consider the digital frontend in Fig. 6. Since we have introduced most of the components in the analog front-end case, computing the power for the digital case is much easier. Notice in the figure, that since beamforming is done in baseband there is no need for phase shifters. Neither do the LNA gains need to compensate for their insertion losses. Therefore, we will use $G_{\mathrm{LNA}}$ instead of $G_{\mathrm{LNA}}^{\mathrm{PS}}$. However, the number of the ADCs is increased to $N_{\mathrm{RX}}$, so is the number of VGAs, mixers and local oscillators. Thus, the DC power drawn by the LNA and VGA is given as

$P_{\mathrm{LNA}}^{\mathrm{DC}}=\frac{G_{\mathrm{LNA}}}{\operatorname{FoM}\left(N_{\mathrm{LNA}}-1\right)}$,

and

$$
\begin{aligned}
G_{\mathrm{VGA}_{\mathrm{dig}}}^{\max }= & P_{\mathrm{BB}}^{\text {out }}-10 \log \left(N_{\mathrm{RX}}\right)+\mathrm{IL}_{\mathrm{mix}} \\
& -\left(G_{\mathrm{LNA}}\right)-P_{\mathrm{RX}}\left(d=d_{\text {max }}\right) .
\end{aligned}
$$

With these, the total power consumed by a fully digital mmWave front-end is

$P_{\mathrm{dig}}=N_{\mathrm{RX}}\left(P_{\mathrm{LNA}}^{\mathrm{DC}}+P_{\mathrm{LO}}+P_{\mathrm{ADC}}+P_{\mathrm{VGA} A_{\mathrm{dig}}}^{\mathrm{DC}}\right)$.

Digital front-end with low-resolution ADCs The front-end here remains the same as in Fig. 6 . The only thing that changes is the resolution $q$ of the ADCs. Notice in Eq. (23), that the power consumption is exponential in $q$. Since the power consumption of a fully digital mmWave frontend grows linearly in the number of antenna elements $N_{\mathrm{RX}}$ as given in Eq. (29), reducing the resolution is the only meaningful way of bringing the power draw of a fully digital front-end. In our calculations below and in Sect. 4 we will show how reducing the quantizer resolution affects both the power drawn by the front-end and the energy consumption during the beam discovery process.

Hybrid front-end For the sake of completeness, we also present the mmWave hybrid front-end in Fig. 7. There are a few options in designing a hybrid front-end. Here, we show the "industry-standard" sub-array architecture where the antenna array is divided by the number of supported digital streams. The depicted frontend can support $M=2$ digital streams each connected to sub-arrays of size $N_{\mathrm{RX}} / 2$. Notice that this circuit has elements from both the fully digital and analog front-ends: $N_{\mathrm{RX}}$ phase shifters and $M=2$ pairs of ADCs. Thus, the total power consumption of the hybrid front-end is

$P_{\text {hy }}=N_{\mathrm{RX}} P_{\mathrm{LNA}}^{\mathrm{DC}}+M\left(P_{\mathrm{LO}}+P_{\mathrm{ADC}}+P_{\mathrm{VGA}}^{\mathrm{DC}}\right)$.

\subsubsection{Front-End Power Consumption}

To give a better picture of each front-end's power consumption, we will now give numerical examples. We will use power draws and losses of individual components as reported in studies in the literature and give a total number for each frontend when put together.

For an LNA with gain $G_{\mathrm{LNA}}=10 \mathrm{~dB}$ with a noise figure of $N_{\mathrm{LNA}}=3 \mathrm{~dB}$ and FoM $=6.5 \mathrm{~mW}^{-1}$ we have used the $90 \mathrm{~nm}$ CMOS LNA reported in ${ }^{7,21,46}$. We have assumed a PS insertion loss ILPS $=10 \mathrm{~dB}$ which the analog front-end LNA gain $G_{\mathrm{LNA}}^{\mathrm{PS}}$ needs to compensate for.

For the ADC, we have used a 4-bit Flashbased ADC reported in ${ }^{36}$ with an FoM of 67.6fJ/conversion step. While the 4-bit ADC falls into the category of a low-resolution ADC, we use the same architecture and FoM for higher quantization of $q=10$ bits for better comparison. For the VGA, we consider the 90nm CMOS reported in $^{50}$. The VGA's FoM is 5280 for an active area of $0.01 \mathrm{~mm}^{2}$. Last, we have assumed an oscillator power draw of $10 \mathrm{dBm}$, a mixer insertion loss of $\mathrm{IL}_{\text {mix }}$ of $6 \mathrm{dBm}$ and baseband power $P_{\mathrm{BB}}^{\text {out }}$ of $10 \mathrm{dBm}$.

Using the above device properties, we, furthermore, consider an operating bandwidth $(B W)$ of $400 \mathrm{MHz}$, the maximum provisioned bandwidth by $3 \mathrm{GPP}$ NR and a maximum distance $d_{\max }$ of 100 meters. We then use the channel model $^{8}$ to calculate the pathloss needed for computing the received power $P_{\mathrm{RX}}$ in Eqs. (24) and (28). Specifically, for an EIRP Tx power of $43 \mathrm{dBm}$ the received power is $-87 \mathrm{dBm}$ at $d_{\max }$ where the channel is expected to be non-line of sight (NLOS). Finally, we assume a sampling rate, $F_{\mathrm{s}}=1 \mathrm{GHz}$ and a receiver array size of $N_{\mathrm{Rx}}=16$. While we assume a system bandwidth of $400 \mathrm{MHz}$, however, according to the NR specifications OFDM sampling rate is set at $491 \mathrm{MHz}$. Furthermore, we consider an oversampling rate at $2 \times$ the system bandwidth to avoid aliasing. Hence, the $1 \mathrm{GHz}$ sampling rate assumption. 


\begin{tabular}{llllll}
\hline \begin{tabular}{l} 
Table 1: mmWave receiver's power consumption $(\mathrm{mW})$ \\
\hline Front-end architecture
\end{tabular} & RFFE & VGA & ADC $(\boldsymbol{q}=10)$ & ADC $(\boldsymbol{q}=4)$ & Total \\
\hline Analog & 257.3 & 1.55 & 133.12 & - & 391.97 \\
Hybrid (M=2) & 267.3 & 3.1 & 266.24 & - & 536.64 \\
Digital (high res.) & 184.7 & 24.8 & 2129.9 & - & 2339.4 \\
Digital (low res.) & 184.7 & 24.8 & - & 33.28 & 242.78 \\
\hline
\end{tabular}

A receiver array size of 16 antennas and a sampling rate of $1 \mathrm{GHz}$ are assumed

By plugging the above numbers into (22)(30), we obtain the front-end power consumption presented in Table 1. With the term RFFE we denote the radio frequency front-end, which is the pre-VGA part of the mmWave front-end, i.e., the LNAs and the LO. We observe a couple of points:

- The power consumption of a high-resolution digital front-end is almost six times the consumption of the analog front-end. However, it is below a factor of $N_{\mathrm{RX}}$. This is because while there are $N_{\mathrm{RX}}$ ADCs in a digital front-end, there are also $N_{\mathrm{RX}}$ phase shifters in an analog one, the insertion loss of which must be compensated by the LNAs. We will see in the next section how the gap between analog and digital closes when we take into account the beam discovery delay.

- Reducing the ADC resolution from $q=10$ to $q=4$ bits has a dramatic effect on the power consumption. It is, in fact, even below that of the analog front-end. However, this reduction in resolution adds a distortion to the signal which will reduce the effective SNR. This reduction has a small effect in the low to median SNR regimes ${ }^{20,21}$.

\section{Evaluation and Simulation}

We now evaluate the performance of our correlation-based detector for receivers performing analog, fully digital, and low-resolution fully digital beamforming. In a series of steps, we first define the simulation setup and parameters, assess the effect of lowering the quantization resolution in fully digital front-ends, and finally illustrate the interplay between the beamspace and the energy consumption of each the frontend architectures presented in Sect. 3.

\subsection{Channel Model and User SNR Distribution}

We consider a single cell of radius $d_{r}=100 \mathrm{~m}$, with the mmWave base station, or gNB, situated at the center. We then randomly drop user equipments in this cell and compute their pathloss to the gNB according to the widely used model $\mathrm{in}^{8}$. This urban multipath model is based on real measurements in $28 \mathrm{GHz}$ bands performed in New York City $^{10,32,43,55}$. While our detector was derived for a single-path LOS channel perfectly aligned with one of the beamspace directions, we will use this multipath channel in our evaluation and simulations. According to this model, links between the user equipment and the gNB are in LOS or NLOS based on an exponential probability distribution parametrized by the distance separating the two, $d$. Close-by user equipments have a high probability of being in LOS while as the distance grows the probability of being in NLOS increases. The resulting omni-directional pathloss, i.e., before applying beamforming, in $\mathrm{dB}$ is computed as

$P_{L}=\mu+10 v \log _{10}(d)+\zeta[\mathrm{dB}], \quad \zeta \sim \mathcal{N}\left(0, \xi^{2}\right)$,

where $\mu, v$ and $\xi^{2}$ are parameters defined by whether the link is LOS or NLOS_-see Table 2.

We then use this pathloss to derive the omnidirectional downlink (DL) SNR at the user equipment, $\mathrm{SNR}_{\text {omni, }}$ as

$\mathrm{SNR}_{\mathrm{omni}}=\frac{P_{\mathrm{RX}}}{N_{0} W_{\text {sys }}}$,

where $P_{\mathrm{RX}}$ is the received omni-directional power resulted from subtracting, in $\mathrm{dB}$ scale, the pathloss in (31) from the transmitted power $P_{\mathrm{TX}}$. Note that all the terms in the equation itself have been converted in a linear scale. The remaining parameters above are the noise power density plus noise figure, $N_{0}$, and the system bandwidth $W_{\text {sys. }}$

Next, we derive the user equipments SNR distribution through 10,000 random drops in our cell and divide them into three regimes: cell
Quantization noise: The difference between the value of the analog signal and its quantized output at the ADC. It can be thought like a rounding error. Thus, the more levels an ADC has the fewer the errors. Lowering the quantization resolution (i.e., levels) results in the increase of this error. The error itself is modeled in our work as a constant degradation in the amplitude plus an additive white Gaussian noise to account for the non-linearity of the quantization process. 


\begin{tabular}{|c|c|c|}
\hline Parameter & Value & Description \\
\hline$d_{r}$ & $100 \mathrm{~m}$ & Cell radius \\
\hline$P_{\mathrm{TX}}$ & $30 \mathrm{dBm}$ & gNB TX power \\
\hline NFUE & $7 \mathrm{~dB}$ & User equipment noise figure \\
\hline$k T$ & $-174 \mathrm{dBm} / \mathrm{Hz}$ & Thermal noise power density \\
\hline$F_{c}$ & $28 \mathrm{GHz}$ & Carrier frequency \\
\hline$W_{\text {sys }}$ & $400 \mathrm{MHz}$ & System bandwidth \\
\hline$P_{\operatorname{LOS}}(d)$ & $\exp \left(-a_{\mathrm{los}} d\right), a_{\mathrm{los}}=67.1 \mathrm{~m}$ & Probability of LOS vs. NLOS \\
\hline$P_{\text {NLOS }}(d)$ & $1-P_{\operatorname{LOS}}(d)$ & Probability of NLOS \\
\hline$P L(d)$ & $\mu+10 v \log 10(d)+\zeta, \zeta \sim \mathcal{N}\left(0, \xi^{2}\right)$ & $\begin{array}{l}\text { Path loss model } \mathrm{dB}, d \text { in } \\
\text { meters }\end{array}$ \\
\hline$\mu \mathrm{LOS}, \nu_{\mathrm{LOS}}, \xi \mathrm{LOS}$ & $\mu=61.4, v=2.0, \xi^{2}=5.8 \mathrm{~dB}$ & LOS parameters \\
\hline$\mu_{\text {NLOS, }}, v_{\text {NLOS }}, \xi_{\text {NLOS }}$ & $\mu=72.0, v=2.92, \xi^{2}=8.7 \mathrm{~dB}$ & NLOS parameters \\
\hline
\end{tabular}

edge user equipments, i.e., the first percentile of the CDF, median users and above, and those in between these regimes. However, in the sequel, we characterize the energy consumed by the analog and digital beamformers during beam discovery for the cell edge and the median users. Figure 8 shows the CDF of the downlink omni-directional SNR distribution, based on Eq. (32), converted in $\mathrm{dB}$ scale. A summary of the parameters and their values used to derive this distribution is presented in Table 2.

\subsection{Low-Resolution Fully Digital Beamforming}

In Sect. 3.2, we presented the power consumption of four mmWave front-ends implementing analog, hybrid, and fully digital beamforming. We also showed that the power consumption of the fully digital front-end could be dramatically reduced by employing low-resolution ADCs.

However, reducing the resolution will degrade the quality of the received signal. We quantify this degradation using the method presented in ${ }^{11,16,24}$. According to $^{24}$, the effect of low-resolution quantization, i.e., analog-to-digital conversion, is modeled as a reverse gain $(1-\gamma)$ multiplied with the received sample if it had gone through an infinite-resolution quantizer and an additive Gaussian noise term. That is if the high quantization sample is given as

$y[n]=x[n]+w[n]$,

where $x[n]$ are the transmitted signal sample and $w[n]$ AWG noise with some variance

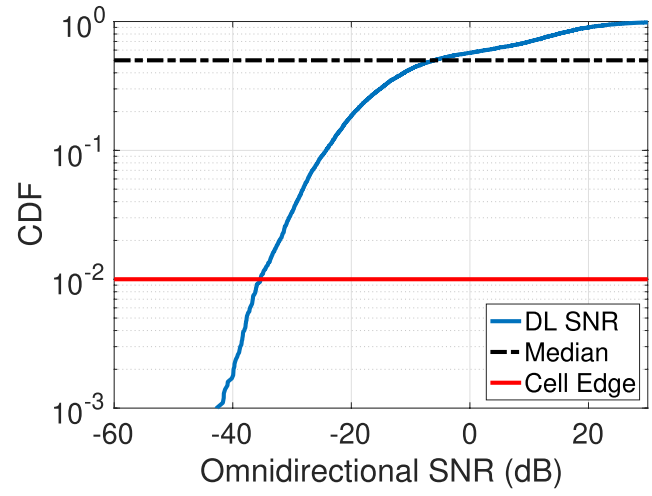

Figure 8: Downlink SNR distribution of 10,000 user equipment drops. Each point corresponds to a value calculated using Eq. (32) and then converted in $\mathrm{dB}$ scale. The solid red line and the dashed gray line denote the cell edge and the median user snr regimes, respectively.

$\mathbb{E}|w[n]|^{2}=\sigma_{n}^{2}$, then the imperfectly quantized received sample is given as

$y_{q}[n]=(1-\gamma) x[n]+(1-\gamma) w[n]+v[n]$.

The last term $v[n]$ is a zero-mean Gausian random variable with the following variance $e^{11,24}$

$\sigma_{\mathrm{q}}^{2}=\mathbb{E}|\nu[n]|^{2}=\gamma(1-\gamma)\left(\mathbb{E}|x[n]|^{2}+\sigma_{n}^{2}\right)$.

Now, using Eq. (33) the effective SNR SNR eff $_{\text {after }}$ quantization is obtained as

$\mathrm{SNR}_{\mathrm{eff}}=\frac{(1-\gamma) \mathrm{SNR}_{\infty}}{1+\gamma \mathrm{SNR}_{\infty}}$, 
where $\mathrm{SNR}_{\infty}$ is the $\mathrm{SNR}$ value of an infinite-resolution quantizer. The value of $\gamma$ depends on the quantizer's design, the input distribution, and the quantization resolution bits $q$. As an example, for a Gaussian input the value of $\gamma$ is only 0.01 .

Thus, we see that low-quantization fully digital beamforming comes with a penalty in the SNR, an observation corroborated by the theoretic work in $^{35,45}$. However, this penalty can be negligible depending on the quantization bits $q$, and the SNR regime, as shown in ${ }^{20,21}$, where the same model was used for OFDM inputs. The effective SNR can be much lower, though, for very high values of $\mathrm{SNR}_{\infty}$ at very low quantization bits, say one or two. However, in cellular systems, the overwhelming majority of user equipments are at low to medium SNR regimes where the distance between the effective $\mathrm{SNR}$ and $\mathrm{SNR}_{\infty}$ is below $3 \mathrm{~dB}$. Furthermore, at a quantization of $q=4$ bits as we consider for our low-resolution fully digital front-end, even at $\mathrm{SNR}_{\infty}$ values as high as $20 \mathrm{~dB}$, this distance is still below $3 \mathrm{~dB}^{20,21}$.

\subsection{System Parameters}

We will compare the mmWave front-ends presented in Sect. 3, within the context of 3GPP NR's PSS discovery. We assume a PSS signal that is confined within one OFDM symbol and 127 sub-carriers. For a sub-carrier spacing of $\Delta_{f}=120 \mathrm{kHz}$, the PSS signal duration and bandwidth are $T_{\mathrm{PSS}}=8.91 \mu \mathrm{s}$ and $W_{\mathrm{PSS}}=15.24 \mathrm{MHz}$, respectively. We will assume that the channel is almost flat within the duration and bandwidth of the signal.

In this work, we assume that during an SS burst, there is no multiplexing with data symbols. That is, all the power of the base station is put on the signals in the SS block. Thus, the omni-directional PSS signal SNR, SNRPSS, is given as

$\mathrm{SNR}_{\mathrm{PSS}}=\frac{P_{\mathrm{RX}} T_{\mathrm{PSS}}}{N_{0}}$.

The above equation is related to the omnidirectional in Eq. (32) as ${ }^{11}$ :

$\mathrm{SNR}_{\mathrm{PSS}}=W_{\mathrm{sys}} \times T_{\mathrm{PSS}} \times \mathrm{SNR}_{\mathrm{omni}}$.

Both the user equipment and the gNB are equipped with uniform planar arrays that allow them to beamsteer in both azimuth (az) and elevation (el). Note that in the sequel, we denote the available directions at the gNB and the user equipment with $N_{D}^{\mathrm{Tx}}$ and $N_{D}^{\mathrm{Rx}}$, respectively. These are the products of $N_{\mathrm{Tx}}$ and $N_{\mathrm{Rx}}$ in each dimension of the 2D arrays. Hence, the beamspace size
$L$ is equal to $N_{D}^{\mathrm{Tx}} \times N_{D}^{\mathrm{Rx}}$. The gNB is assumed to perform only hybrid beamforming with $M=2$ directions at a time, while the user equipment may employ one of analog, fully digital, and lowresolution fully digital beamforming. Note that we keep the transmission power of the gNB fixed at $30 \mathrm{dBm}$ (Table 2). Therefore, transmitting the PSS in $M=2$ directions implies that the sum of the power transmitted in each direction must be $30 \mathrm{dBm}$. We will assume equal power transmission. Thus, the signal in each direction will carry half of the total $30 \mathrm{dBm}$ power. The resulting beamforming gains will be applied to the user equipments' omni-directional PSS signal SNR given by Eq. (36) or to its corresponding effective SNR in case of the low-resolution digital front-end.

Also, we assume a beamspace discovery process, as described in Sect. 2.2.1, where the gNB transmits the PSS directionally in its $N_{D}^{\mathrm{Tx}}$ direction within an SS burst of duration 5 ms every $T=20 \mathrm{~ms}$. The user equipment also sweeps its $N_{D}^{\mathrm{Rx}}$ directions in search of one of the $N_{\mathrm{PSS}}=3$ PSS sequences. Finally we compute the threshold $\theta$ in test (10). We will do so through the false alarm probability $P_{\mathrm{FA}}$. Suppose we would like to maintain a constant target false alarm rate of $R_{\mathrm{FA}}$ during each searching period. Then, the false alarm probability and the false alarm rate are connected through

$P_{\mathrm{FA}}=\frac{R_{\mathrm{FA}}}{N_{\mathrm{PSS}} N_{\mathrm{dly}} N_{\mathrm{FO}}}$.

Now, $N_{\text {dly }}$ is the number of delay hypotheses. That is at which delay tau the signal was present. Since the PSS transmission is periodic, the number of the delay hypotheses is bounded, and it is the number of the samples within each SS burst period. If the signal was present at a delay $\tau_{1}$, then it is also present at delay $T+\tau_{1}$, where $T$ is the SS burst period.

To compensate for two other sources of uncertainty, we incorporate them into the $P_{\mathrm{FA}}$ calculation. These are the number of possible PSS sequences $N_{\text {PSS }}$ and the number of frequency offsets hypotheses $N_{\text {FO }}$. There are $N_{\text {PSS }}$ sequences that the user equipment may mistake one with the other two and are factored in the false alarm calculation. Next, we approximate $N_{\text {FO }}$ as follows. First, we assume a local oscillator error of \pm 10 parts per million (ppm) and a Doppler shift for a velocity up to $30 \mathrm{~km} / \mathrm{h}$ at $F_{\mathrm{c}}=28 \mathrm{GHz}$. The LO error and the Doppler shift will define a range $\pm \Delta_{f \text { max }}$. We then discretize this range so that in each interval the channel does not rotate more
False alarm rate: The amount of false positives during a pre-determined time interval. Often system procedures are designed with a certain target false alarm rate they tolerate. 
Miss detection probability: The probability of missing the signal when it is present.

\begin{tabular}{|c|c|}
\hline Parameter & Value \\
\hline Total system bandwidth, $W_{\text {sys }}$ & $400 \mathrm{MHz}$ \\
\hline Signal duration, TPSS & $8.91 \mu \mathrm{s}(1$ OFDM symbol) \\
\hline Sub-carrier spacing, $\Delta_{\mathrm{f}}$ & $120 \mathrm{kHz}$ \\
\hline PSS Bandwidth, WPSS & 15.24 MHz (127 sub-carriers) \\
\hline SS burst period, $T$ & $20 \mathrm{~ms}$ \\
\hline SS burst duration, $T_{\text {ssb }}$ & $5 \mathrm{~ms}$ \\
\hline Total false alarm rate per scan cycle, $R_{\mathrm{FA}}$ & 0.01 \\
\hline Number of PSS waveform hypotheses NPSS & 3 \\
\hline Number of frequency offset hypotheses, $N_{F O}$ & 3 \\
\hline gNB antenna & $N_{T X} \times N_{T X}$ uniform planar array \\
\hline UE antenna & $N_{\mathrm{RX}} \times N_{\mathrm{RX}}$ uniform planar array \\
\hline gNB antenna elements in each dimension & $N_{\text {TX }}=8$ \\
\hline UE antenna elements in the azimuth dimension & Varies: $N_{\mathrm{RX}}^{\mathrm{az}}=2,4$ \\
\hline UE antenna elements in the elevation dimension & Varies: $N_{R X}^{e l}=2,4$ \\
\hline gNB directions & $N_{D}^{T X}=N_{T X}^{\mathrm{az}} \times N_{T X}^{\mathrm{e}}=8 \times 8=64$ \\
\hline UE directions & varies $N_{D}^{\mathrm{RX}}=N_{\mathrm{RX}}^{\mathrm{az}} \times N_{\mathrm{RX}}^{\mathrm{el}}=4,8,16$ \\
\hline gNB beamforming & Hybrid $M=2$ \\
\hline UE beamforming & $\begin{array}{l}\text { Varied: analog, digital, low-res. } \\
\text { digital }\end{array}$ \\
\hline
\end{tabular}

than $\pi / 4$ within the duration of one OFDM symbol. The number of frequency offset hypotheses is then the number of obtained intervals.

The threshold $\theta$, therefore, is the value of the normalized correlation $\rho_{k \ell}$ which corresponds to

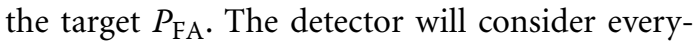
thing above this threshold as received signal and anything below as noise.

All the parameters described above are tabulated in Table 3.

\subsection{Comparison of Different Beamforming Architectures}

Now that we have defined the channel model, system and signal parameters, and described the beamspace and beam discovery procedure, we can start the comparison between the mmWave front-ends presented in Sect. 3.2. We first look into the beam discovery delays with beamsweeping for the beamformers and then tie these delays to the power consumptions reported in Sect. 3.2.2.

\subsubsection{Beamsweeping Delay}

We estimate the initial discovery delay as the time needed to go through all the beams in the beamspace times the total number beamsweeps $K$ necessary to determine the best direction for a target miss detection probability $P_{\mathrm{MD}}^{\mathrm{tgt}}$. Hence, $K$ is a function of $P_{\mathrm{MD}}^{\mathrm{tgt}}$. Note that $K$ is also a function of the SNR. A low SNR user at the cell edge will have to sweep the beamspace and visit the same correct direction multiple times to accumulate enough energy required for the signal detection.

In Fig. 9 we present the miss detection probability $P_{\text {MD }}$ vs SNRPSS, i.e., the PSS signal SNR, . There, we show the number of beamsweeps necessary to discover the correct path to the $\mathrm{gNB}$ for a $P_{\mathrm{MD}}^{\mathrm{tgt}}=1 \%$. A mobile user at the cell edge needs $K=3$ beamsweeps to get the right direction, while the rest of the users can detect the signal in one beamsweep. Take as an example the case of the analog receiver with $N_{D}^{\mathrm{Rx}}=16$ directions, while the transmitter has $N_{D}^{\mathrm{Tx}}=64$ directions. The size $L$ of the beamspace is then $16 \times 64=1024$. Then, the delay in initial discovery $T_{\text {delay }}\left(P_{\mathrm{MD}}^{\mathrm{tgt}}\right)$, for such a user equipment to successfully discover the true direction is 


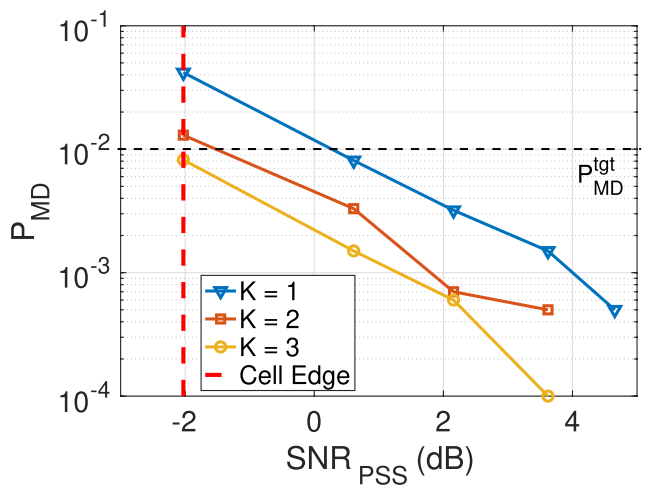

Figure 9: Analog receiver: miss detection probability $P_{\mathrm{MD}}$ vs SNRPss in $\mathrm{dB}$, as given by Eq. (36). $K=1,2,3$ and target miss detection probability $P_{\mathrm{MD}}^{\mathrm{tgt}}=1 \%$

Table 4: Upper bounds of initial discovery delays for different mmWave front-ends.

\begin{tabular}{lll}
\hline Front-end architecture & $\begin{array}{l}\text { Cell edge } \\
\text { discovery } \\
\text { delay }\end{array}$ & $\begin{array}{l}\text { Median user } \\
\text { discovery } \\
\text { delay }\end{array}$ \\
\hline Analog & $960 \mathrm{~ms}$ & $320 \mathrm{~ms}$ \\
\hline Digital (high res.) & $60 \mathrm{~ms}$ & $20 \mathrm{~ms}$ \\
Digital (low res.) & $80 \mathrm{~ms}$ & $20 \mathrm{~ms}$ \\
\hline
\end{tabular}

$P_{\mathrm{MD}}^{\text {tgt }}$ is set to $1 \%$

$$
\begin{aligned}
& \frac{\left(K\left(P_{\mathrm{MD}}^{\mathrm{tgt}}\right)-1\right) \times L \times T}{N_{\mathrm{D}}^{\mathrm{Tx}}}+T_{\mathrm{ssb}} \\
& \leq T_{\text {delay }}\left(P_{\mathrm{MD}}^{\mathrm{tgt}}\right) \leq \frac{K\left(P_{\mathrm{MD}}^{\mathrm{tgt}}\right) \times L \times T}{N_{\mathrm{D}}^{\mathrm{Tx}}} \\
& \quad=\frac{20480 K\left(P_{\mathrm{MD}}^{\mathrm{tgt}}\right)}{64} \mathrm{~ms},
\end{aligned}
$$

where $T=20 \mathrm{~ms}$ is the SS burst period and $T_{\mathrm{ssb}}=5 \mathrm{~ms}$ the duration of an SS burst. The reason we divided the quantity above with $N_{D}^{\mathrm{Tx}}$ is that within each SS burst period $T$, the gNB sweeps all its directions in one SS burst. Also, notice that we did not divide $L$ by the number of the RF chains $M=2$ at the transmitter. Since the NR procedure defines a maximum of $B=32$ SS blocks within one SS burst and the number of directions the gNB scans is $N_{\mathrm{D}}^{\mathrm{Tx}}=64$, the reduction in the effective beamspace due to hybrid beamforming is accounted for. Otherwise, dividing Eq. (38) by $M$ would imply that the user equipment probes $M=2$ directions each time, which is not correct since it employs an analog beamformer.
From Eq. (38), we can see that even for a user that is not at the cell edge, i.e., $K\left(P_{\mathrm{MD}}^{\mathrm{tgt}}\right)=1$, the initial discovery delay can go as high as $320 \mathrm{~ms}$, when the entire control-plane delay of $4 \mathrm{G}$ LTE must be below $50 \mathrm{~ms}^{1}$. For the cell edge user, on the other hand, this delay is between $640 \mathrm{~ms}$ and almost a second. This highlights the large impact of the beamspace size on the initial discovery delay. Now, during initial discovery, the user equipment must be constantly "on" searching for the correct beamspace. This amount of delay, as we will show next, has a large impact on the power consumption of the analog beamformer.

In Table 4 following the same process and using Eq. (38), we give the upper bounds of the initial discovery delay for the other two kinds of front-ends, fully digital and low-resolution fully digital. All the numbers are for a user equipment array size of 16 elements. Notice that the SNR penalty due to low quantization is negligible in both the cell edge and the rest of the area. The large gain compared to analog was the result of shrinking the beamspace size $L$ to only $N_{\mathrm{D}}^{\mathrm{Tx}}$ which is all covered in one SS burst.

\subsubsection{Beamsweeping Energy Consumption}

We have so far characterized the beam discovery delay of the analog, the high-resolution digital, and the low-resolution digital beamformers. The energy consumption of a front-end is tightly connected to the amount of time it needs to operate. Since the user equipment is assumed to be always "on" trying to attach to a gNB, it is necessary to go beyond the device power consumption and look at energy consumption during the time needed to establish a directional link between the user equipment and the gNB. In Fig. 10, we show the energy consumption of the three frontends, where the user equipment array size is $N_{\mathrm{RX}}=16$. The difference between analog and the two versions of digital is astonishing. The energy consumption of analog exceeds even that of highresolution digital. Remember, when we looked at the front-ends' power consumption (Joules/s) in Sect. 3.2.2, the consumption of digital beamforming was six times that of analog beamforming. But, as Fig. 10 establishes, the analog beamforming front-end burns almost four times more energy than even the high-resolution digital front to compensate for the "spatial blindness" during the beam sweep procedure.

However, since after establishing the directional link, in the data communication phase, analog is expected to burn less power than fully digital, we believe that mmWave systems will
Control-plane: The set of signals and procedures pertaining to network functionalities such as network access, mobility management, security authentication, and routing. 


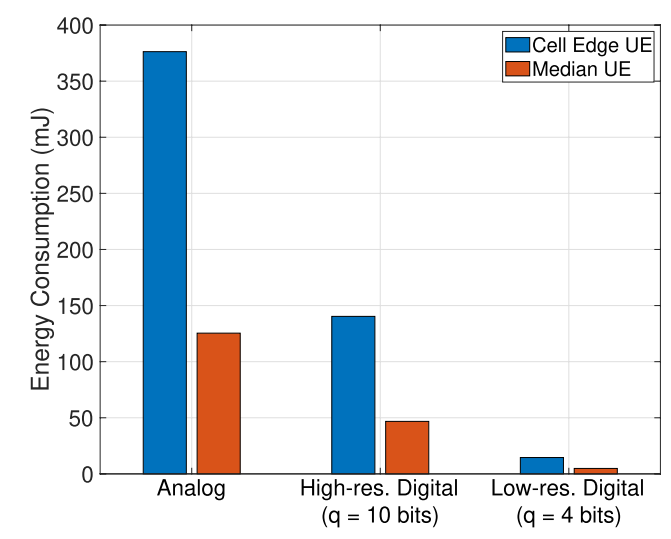

Figure 10: Energy consumption of the mmWave front-ends: analog, high-resolution digital and low-resolution digital. Number of antenna elements at the user equipment $N_{R x}=16$. Cell edge user equipment in blue, median user equipment in red.

need to use a low-resolution fully digital frontend; despite the fact that the difference in energy consumption between high- and low-resolution digital front-ends is quite small during beam discovery.

Next, we turn our attention to the number of antenna elements at the receiver. We would like to know how the energy consumption scales with the size of the antenna array in the initial beamsweeping phase. In other words, what is the impact of various beamspace sizes on energy consumption? Fig. 11 answers this question. We try three different configurations at the user equipment: a $2 \mathrm{D}$ array of size 4 , a $2 \mathrm{D}$ array of size 8 , and a $2 \mathrm{D}$ array of size 16 . There are a few interesting points these two figures raise:

- First, while fewer antennas do bring the energy consumption of the user equipment down, analog beamforming still burns considerably more energy than both the digital front-ends.

- Second, the gap between analog and digital beamforming widens as more antennas are employed at the user equipment. The reason is the following: when the number of antennas is small, and the post-BF SNR is low, analog beamforming looks into fewer directions at each period. This means that the most crucial factor in the front-ends' energy consumption is the size of the beamspace. This leads to higher beam discovery delays resulting in longer "on" time for the user equipment. This is more evident in Fig. 11b where all front-

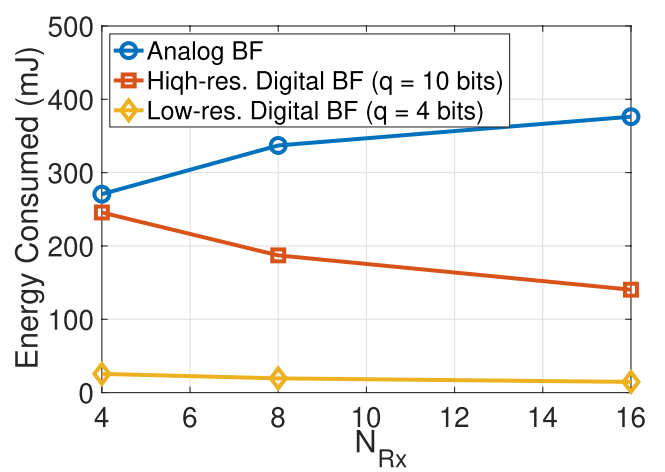

(a) Cell Edge user equipment

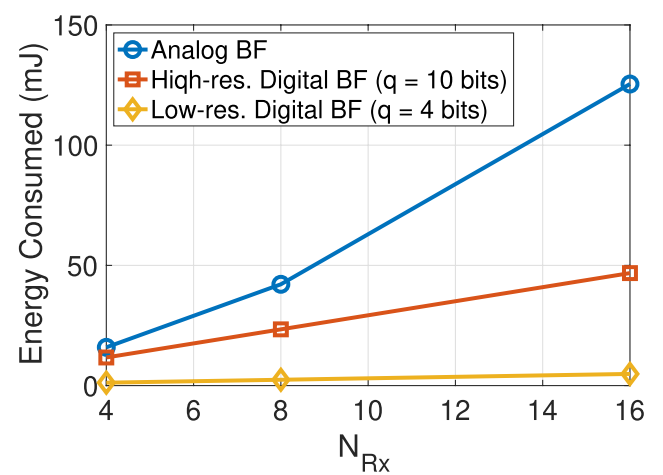

(b) Median user equipment

Figure 11: Energy consumption as a function of the user equipment array size. a Energy con-

sumption at the cell edge. b Energy consumption of the median user equipment.

ends need no more than $K=1$ beamsweep to detect the true path to the gNB.

- Related to the previous point, the value of digital beamforming is evident in every antenna configuration. The reduction in the size of the effective beamspace is the fundamental and necessary aspect of expediting beam discovery and, subsequently, lowering energy consumption. Beyond initial link establishment, digital beamforming will reduce the complexities involved in handovers and avoiding blockage in connected mode.

\section{Conclusion and Future Directions}

The realization of mmWave communication systems requires addressing two crucial challenges, namely beam discovery delay and energy consumption. In this work, we have revealed how closely these two are tied together. Specifically, we showed that employing analog beamforming can significantly increase both the 
discovery delay and, subsequently, energy consumption. Hence, analog beamforming does not buy us lower energy consumption.

Digital beamforming, on the other hand, achieves lower delays and lower energy consumption during beam discovery. In addition, depending on operating SNR, using digital beamforming can allow the use of multi-stream communication and allow joint, flexible scheduling of frequency resources and directional beams. This may be extremely useful in the case of small data packets. By expediting beam discovery, fully digital may enable more aggressive use of sleep mode since beam tracking and paging can be less frequent. In fact, studying the effect of beamforming architectures on higher layers, i.e., MAC and TCP/IP, in a multicell setup is an interesting direction for future research.

It is true that when the directional data link is established, analog burns less energy compared to fully digital. Reducing the quantization resolution of the ADC pairs, however, can bring the energy requirements in data communication at the level of analog beamforming at a penalty negligible in most SNR regimes. Thus, low-resolution digital beamforming enables the realization of mmWave cellular systems with low power, low control latency, and more flexible data scheduling.

\section{Publisher's Note}

Springer Nature remains neutral with regard to jurisdictional claims in published maps and institutional affiliations.

\section{Open Access}

This article is licensed under a Creative Commons Attribution 4.0 International License, which permits use, sharing, adaptation, distribution and reproduction in any medium or format, as long as you give appropriate credit to the original author(s) and the source, provide a link to the Creative Commons licence, and indicate if changes were made. The images or other third party material in this article are included in the article's Creative Commons licence, unless indicated otherwise in a credit line to the material. If material is not included in the article's Creative Commons licence and your intended use is not permitted by statutory regulation or exceeds the permitted use, you will need to obtain permission directly from the copyright holder. To view a copy of this licence, visit http://creativecommons.org/ licenses/by/4.0/.
Received: 4 December 2019 Accepted: 20 March 2020

Published online: 4 May 2020

\section{References}

1. 3GPP (2010) LTE - Requirements for further advancements for Evolved Universal Terrestrial Radio Access (E-UTRA) (LTE-Advanced) - Rel. 9. TR 36.913

2. 3GPP (2018) NR - Physical channels and modulation - Rel. 15. TS 38.211

3. 3GPP (2017) NR - Physical layer measurements Rel. 15. TS 38.215

4. 3GPP (2018) NR - Physical layer procedures for control — Rel. 15. TS 38.213

5. 3GPP (2018) NR - Radio Resource Control (RRC); Protocol specification - Rel. 15. TS 38.331

6. Abbas WB, Zorzi M (2016) Context information based initial cell search for millimeter wave $5 \mathrm{G}$ cellular networks. In: European conference on networks andcommunications (EuCNC). IEEE, pp 111-116

7. Adabi E, Heydari B, Bohsali M, Niknejad AM (2007) 30 $\mathrm{GHz}$ CMOS low noise amplifier. In: 2007 IEEE radio frequency integrated circuits (RFIC)symposium, pp 625-628

8. Akdeniz M, Liu Y, Samimi M, Sun S, Rangan S, Rappaport T, Erkip E (2014) Millimeter wave channel modeling and cellular capacity evaluation. IEEE J Sel Areas Commun 32(6):1164-1179

9. Alkhateeb A, Nam Y, Rahman MS, Zhang J, Heath RW (2017) Initial beam association in millimeter wave cellular systems: analysis and design insights. IEEE Trans Wirel Commun 16(5):2807-2821

10. Azar Y et al (2013) $28 \mathrm{GHz}$ propagation measurements for outdoor cellular communications using steerable beam antennas in New York city. In: 2013 IEEEinternational conference on communications (ICC), pp 5143--5147

11. Barati CN, Hosseini S, Rangan S, Liu P, Korakis T, Panwar S, Rappaport TS (2015) Directional cell discovery in millimeter wave cellular networks. IEEE Trans Wirel Commun 14(12):6664-6678

12. Barati CN, Hosseini SA, Mezzavilla M, Korakis T, Panwar SS, Rangan S, Zorzi M (2016) Initial access in millimeter wave cellular systems. IEEE Trans Wirel Commun 15(12):7926-7940

13. Booth MB, Suresh V, Michelusi N, Love DJ (2019) Multi-armed bandit beam alignment and tracking for mobile millimeter wave communications. IEEE Commun Lett 23(7):1244-1248

14. Capone A, Filippini I, Sciancalepore V (2015) Context information for fast cell discovery in $\mathrm{mm}$-wave $5 \mathrm{G}$ networks. In: Proceedings of European wireless 2015;21th european wireless conference, pp 1-6

15. Capone A, Filippini I, Sciancalepore V, Tremolada D (2015) Obstacle avoidance cell discovery using mmwaves directive antennas in 5G networks. In: 2015 
IEEE26th annual international symposium on personal, indoor, and mobile radio communications (PIMRC), pp 2349-2353

16. Chen JH, Gersho A (1987) Gain-adaptive vector quantization with application to speech coding. IEEE Trans Commun COM-35(9):918-930

17. Chiu S, Ronquillo N, Javidi T (2019) Active learning and csi acquisition for mmwave initial alignment. IEEE J Sel Areas Commun 37(11):2474-2489

18. De Donno D, Palacios J, Widmer J (2017) Millimeterwave beam training acceleration through low-complexity hybrid transceivers. IEEE Trans Wirel Commun 16(6):3646-3660

19. Devoti F, Filippini I, Capone A (2018) Mm-wave initial access: a context information overview. In: 2018 IEEE 19th international symposium on "A World of Wireless, Mobile and Multimedia Networks" (WoWMoM), pp 1-9

20. Dutta S, Barati CN, Dhananjay A, Rangan S (2017) 5G millimeter wave cellular system capacity with fully digital beamforming. In: 2017 51st Asilomar conference on signals, systems, and computers, pp 1224-1228

21. Dutta S, Barati CN, Ramirez D, Dhananjay A, Buckwalter JF, Rangan S (2020) A case for digital beamforming at mmwave. IEEE Trans Wirel Commun 19(2):756-770

22. Eliasi PA, Rangan S, Rappaport TS (2017) Low-rank spatial channel estimation for millimeter wave cellular systems. IEEE Trans Wirel Commun 16(5):2748-2759

23. Filippini I, Sciancalepore V, Devoti F, Capone A (2018) Fast cell discovery in mm-wave $5 \mathrm{~g}$ networks with context information. IEEE Trans Mob Comput 17(7):1538-1552

24. Fletcher AK, Rangan S, Goyal VK, Ramchandran K (2007) Robust predictive quantization: analysis and design via convex optimization. IEEE J Sel Top Signal Process 1(4):618-632

25. Garcia GE, Seco-Granados G, Karipidis E, Wymeersch H (2018) Transmitter beam selection in millimeter-wave mimo with in-band position-aiding. IEEE Trans Wirel Commun 17(9):6082-6092

26. Giordani M, Polese M, Roy A, Castor D, Zorzi M (2019) A tutorial on beam management for $3 \mathrm{~g} \mathrm{pp} \mathrm{nr}$ at mmwave frequencies. IEEE Commun Surv Tutor 21(1):173-196

27. Guo H, Makki B, Svensson T (2017) A genetic algorithmbased beamforming approach for delay-constrained networks. In: 2017 15th international symposium on modeling and optimization in mobile, ad hoc, and wireless networks (WiOpt). pp 1-7

28. Hashemi M, Sabharwal A, Emre Koksal C, Shroff NB (2018) Efficient beam alignment in millimeter wave systems using contextual bandits. In: IEEEINFOCOM 2018 - IEEE conference on computer communications, pp 2393-2401

29. Hussain M, Love DJ, Michelusi N (2017) Neyman-Pearson codebook design for beam alignment in millimeterwave networks. In: 1st ACM Workshop on MillimeterWave Networks and Sensing Systems 2017 (mmNets '17). ACM, pp 17-22
30. IEEE (2016) IEEE Standard for Information technology-Telecommunications and information exchange between systems Local and metropolitan area networksSpecificrequirements - Part 11: Wireless LAN Medium Access Control (MAC) and Physical Layer (PHY) Specifications. In: IEEE Std 802.11-2016 (Revision of IEEEStd 802.11-2012), pp 1-3534

31. Jaesim A, Siasi N, Aldalbahi A, Ghani N (2019) Beambundle codebook for highly directional access in mmwave cellular networks. IEEE Commun Lett 23(11):2104-2108

32. MacCartney GR, Samimi MK, Rappaport TS (2015) Exploiting directionality for millimeter-wave system improvement. In: 2015 IEEE internationalconference on communications (ICC), pp 2416-2422

33. Marandi MK, Rave W, Fettweis G (2019) Beam selection based on sequential competition. IEEE Signal Process Lett 26(3):455-459

34. Marcano AS, Christiansen HL (2016) Macro cell assisted cell discovery method for 5G mobile networks. In: 2016 IEEE 83rd vehicular technology conference (VTC Spring).pp 1-5

35. Mo J, Heath RW (2015) Capacity analysis of one-bit quantized MIMO systems with transmitter channel state information. IEEE Trans Signal Process 63(20):5498-5512

36. Nasri B, Sebastian SP, You KD, RanjithKumar R, Shahrjerdi D (2017) A $700 \mu \mathrm{W}$ 1GS/s 4-bit folding-flash ADC in $65 \mathrm{~nm}$ CMOS for wideband wirelesscommunications. In: 2017 IEEE International Symposium on Circuits and Systems (ISCAS), pp 1-4

37. Palacios J, De Donno D, Giustiniano D, Widmer J (2016) Speeding up mmwave beam training through low-complexity hybrid transceivers. In: 2016 IEEE 27th annual international symposium on personal, indoor, and mobile radio communications (PIMRC). pp 1-7

38. Park E, Choi, Y, Han Y (2017) Location-based initial access and beam adaptation for millimeter wave systems. In: 2017 IEEE wireless communications and networking conference (WCNC). pp 1-6

39. Yavuz M (2018) How will 5G transform industrial IoT? Qualcomm Technologies Inc, San Diego, CA, USA

40. Raghavan V, Cezanne J, Subramanian S, Sampath A, Koymen O (2016) Beamforming tradeoffs for initial ue discovery in millimeter-wave mimo systems. IEEE J Sel Top Signal Process 10(3):543-559

41. Rappaport TS (2002) Wireless communications: principles and practice, 2nd edn. Prentice Hall, Upper Saddle River

42. Rave W, Khalili-Marandi M (2019) The elimination game or: beam selection based on M-ary sequential competition \& elimination. In: WSA 2019; 23rd international ITG workshop on smart antennas, pp 1-8

43. Samimi $\mathrm{M}$ et al (2013) $28 \mathrm{GHz}$ angle of arrival and angle of departure analysis for outdoor cellular communications using steerable beam antennas in New York City. In: 
P013 IEEE 77th Vehicular Technology Conference (VTC Spring), pp 1-6

44. Sim GH, Klos S, Asadi A, Klein A, Hollick M (2018) An online context-aware machine learning algorithm for $5 \mathrm{~g}$ mmwave vehicular communications. IEEE/ACM Trans Netw 26(6):2487-2500

45. Singh J, Dabeer O, Madhow U (2009) On the limits of communication with low-precision analog-to-digital conversion at the receiver. IEEE Trans Commun 57(12):3629-3639

46. Song I, Jeon J, Jhon H, Kim J, Park B, Lee JD, Shin H (2008) A simple figure of merit of RF MOSFET for low-noise amplifier design. IEEE Electron Device Lett 29(12):1380-1382

47. Song X, Haghighatshoar S, Caire G (2018) A scalable and statistically robust beam alignment technique for millimeter-wave systems. IEEE Trans Wirel Commun 17(7):4792-4805

48. Souto VDP, Souza RD, Uchoa-Filho BF, Li Y (2019) A novel efficient initial access method for $5 \mathrm{~g}$ millimeter wave communications using genetic algorithm. IEEE Trans Veh Technol 68(10):9908-9919

49. Van Trees HL (2001) Detection, estimation and modulation theory, part I. Wiley, New York
50. Wang Y, Afshar B, Ye L, Gaudet VC, Niknejad AM (2012) Design of a low power, inductorless wideband variablegain amplifier for high-speed receiver systems. IEEE Trans Circuits Syst I Regul Pap 59(4):696-707

51. Xiu Y, Wu J, Xiu C, Zhang Z (2019) Millimeter wave cell discovery based on out-of-band information and design of beamforming. IEEE Access 7:23076-23088

52. Yan H, Cabria D (2016) Compressive sensing based initial beamforming training for massive MIMO millimeterwave systems. In: 2016 IEEE global conference on signal and information processing (GlobalSIP), pp 620-624

53. Zhang J, Huang Y, Shi Q, Wang J, Yang L (2017) Codebook design for beam alignment in millimeter wave communication systems. IEEE Trans Commun 65(11):4980-4995

54. Zhang Q, Saad W, Bennis M, Debbah M (2016) Quantum game theory for beam alignment in millimeter wave device-to-device communications. In: 2016 IEEE global communications conference (GLOBECOM), pp 1-6

55. Zhao $\mathrm{H}$ et al (2013) $28 \mathrm{GHz}$ millimeter wave cellular communication measurements for reflection and penetration loss in and around buildings in New York City. In: 2013 IEEE International Conference on Communications (ICC), pp 5163-5167 


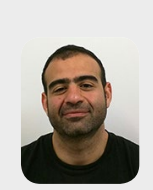

C. Nicolas Barati received the Engineering Diploma degree in computer, telecommunications, and network engineering from the University of Thessaly, Volos, Greece, in 2011, and the Ph.D. degree in electrical engineering from NYU Tandon School of Engineering, Brooklyn, NY, USA, in 2018. He is currently a Post-Doctoral Research Associate with Rice University, Houston, TX, USA. His research interests include wireless communications, massive MIMO, mmWave, and wireless MAC protocols.

Sourjya Dutta received his B.Tech from the National Institute of Technology, Durgapur, in 2012 in Electronics and Communications Engineering. He received his Ph.D. from New York York University in 2020 in Electrical Engineering. He is currently with Qualcomm Technologies Inc., San Diego, as a senior systems engineer. His research interests are in wireless communications, signal processing, and millimeterwave technologies.

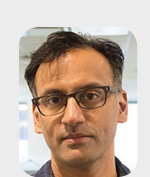

Dr. Sundeep Rangan received a B.A.Sc. at the University of Waterloo, Canada, and an M.Sc. and Ph.D. at the University of California, Berkeley, all in Electrical Engineering. $\mathrm{He}$ has held postdoctoral appointments at the University of Michigan, Ann Arbor, and Bell Labs. In 2000, he co-founded (with four others) Flarion Technologies, a spin-off of Bell Labs, that developed Flash OFDM, the first cellular OFDM data system and pre-cursor to $4 \mathrm{G}$ cellular systems including LTE and WiMAX. In 2006, Flarion was acquired by Qualcomm Technologies. Dr. Rangan was a Director of Engineering at Qualcomm involved in OFDM infrastructure products. He joined the ECE department at NYU Tandon (formerly NYU Polytechnic) in 2010. He is a Fellow of the IEEE and the
Associate Director of NYU WIRELESS, an industry-academic research center on next-generation wireless systems.

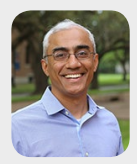

Ashutosh Sabharwal is the Department Chair and Earnest Dell Butcher Professor in the Department of Electrical and Computer Engineering, Rice University. He currently works in two research areas. His first area of research is wireless. He is the founder of the WARP project (warp.rice.edu), an opensource project which is now in use at more than 125 research groups worldwide, and has been used by more than 500 research articles. He is currently leading RENEW (renewwireless.org ), an NSF PAWR project, that is developing open-source massive MIMO platform for a deployed cityscale national wireless testbed. He is also co-leading the new ARL-Rice Center for advanced materials and networks. His second area of research focuses on health, at the intersection of engineering, behavioral sciences, and medicine, and established Scalable Health Labs (sh.rice.edu ). Scalable Health Labs' mission is to develop methods to uncover behavior-biology causal pathways, with a specific focus on three areas: bio-behavioral sensing, mobile bio-imaging, and data science methodologies. Sabharwal is leading several NSF-funded center-scale proposals; most notably, he is the PI of "See below the skin" (seebelowtheskin.org) for noninvasive bio-imaging multi-university effort, and co-investigator of NSF Engineering Research Center, PATHS-UP (pathsup.org) that is developing cost-effective personalized technologies for under-served populations. His research has led to four commercial spinoffs (one in wireless and three in healthcare). He is an IEEE Fellow and was awarded the 2017 IEEE Jack Neubauer Memorial Award, 2018 IEEE Advances in Communications Award, 2019 ACM Sigmobile Test-oftime Award and 2019 ACM MobiCom Community Contributions Award. 